\title{
Visualization formats of patient-reported outcome measures in clinical practice: a systematic review about preferences and interpretation accuracy
}

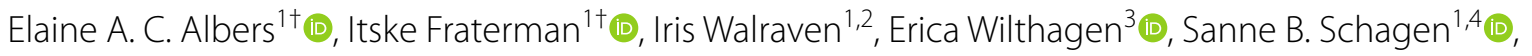
Iris M. van der Ploeg ${ }^{5}$, Michel W. J. M. Wouters ${ }^{5,6}{ }^{\mathbb{C}}$, Lonneke V. van de Poll-Franse ${ }^{1,7,8} \mathbb{C}$ and Kelly M. de Ligt ${ }^{*}$ (D)

\begin{abstract}
Purpose: The use of Patient-Reported Outcome Measures (PROMs) for individual patient management within clinical practice is becoming increasingly important. New evidence about graphic visualization formats for PROMs scores has become available. This systematic literature review evaluated evidence for graphic visualization formats of PROMs data in clinical practice for patients and clinicians, for both individual and group level PROMs data.

Methods: Studies published between 2000 and 2020 were extracted from CINAHL, PubMed, PsychInfo, and Medline. Studies included patients $\geq 18$ years old in daily clinical practice. Papers not available in English, without full-text access, or that did not specifically describe visualization of PROMs data were excluded. Outcomes were: visualization preferences; interpretation accuracy; guidance for clinical interpretation.

Results: Twenty-five out of 789 papers were included for final analysis. Most frequently studied formats were: bar charts, line graphs, and pie charts. Patients preferred bar charts and line graphs as these were easy and quick for retrieving information about their PROMs scores over time. Clinicians' interpretation accuracy and preferences were similar among graphic visualization formats. Scores were most often compared with patients' own previous scores; to further guide clinical interpretation, scores were compared to norm population scores. Different 'add-ons' improved interpretability for patients and clinicians, e.g. using colors, descriptions of measurement scale directionality, descriptive labels, and brief definitions.
\end{abstract}

Conclusion: There was no predominant graphical visualization format approach in terms of preferences or interpretation accuracy for both patients and clinicians. Detailed clarification of graph content is essential.

Keywords: Patient reported outcome measures, Data visualization, Shared decision-making, Systematic review

\footnotetext{
*Correspondence: K.d.ligt@nki.nl

${ }^{\dagger}$ Elaine A. C. Albers and Itske Fraterman have contributed equally to this work

${ }^{1}$ Department of Psychosocial Research, Division of Psychosocial Research and Epidemiology, The Netherlands Cancer Institute, Amsterdam, The Netherlands

Full list of author information is available at the end of the article
}

(c) The Author(s) 2022. Open Access This article is licensed under a Creative Commons Attribution 4.0 International License, which permits use, sharing, adaptation, distribution and reproduction in any medium or format, as long as you give appropriate credit to the original author(s) and the source, provide a link to the Creative Commons licence, and indicate if changes were made. The images or other third party material in this article are included in the article's Creative Commons licence, unless indicated otherwise in a credit line to the material. If material is not included in the article's Creative Commons licence and your intended use is not permitted by statutory regulation or exceeds the permitted use, you will need to obtain permission directly from the copyright holder. To view a copy of this licence, visit http://creativecommons.org/licenses/by/4.0/. 


\section{Plain English summary}

Patient-Reported Outcome Measures (PROMs) capture patients'self-reported health through the use of questionnaires. PROMs measure health related quality of life, daily functioning, and symptom experience, which are becoming increasingly important to incorporate in clinical practice for individual patient management. To present PROMs within clinical practice, raw or summarized PROMs scores can be visualized in graphical formats. To be useful during clinical encounters, both patients and clinicians ought to interpret such formats correctly. New evidence about graphic visualization formats for PROMs scores has become available. Therefore, we systematically reviewed the literature to evaluate evidence for graphic visualization formats of PROMs data in clinical practice. In 25 included papers, most studies used graphical formats like bar charts, line graphs, and pie charts for presenting PROMs scores. There was no predominant graphical visualization format approach in terms of preferences or interpretation accuracy for both patients and clinicians. Patients preferred bar charts and line graphs as these were easy and quick for retrieving information about their PROMs scores over time. Clinicians' interpretation accuracy and preferences were similar among graphic visualization formats. The graphical interpretation of PROMs data for patients and clinicians can be improved by using colors, descriptions of measurement scale directionality, descriptive labels, and brief definitions.

\section{Introduction}

With an increasing emphasis on patient-centred care, there is a growing interest in outcome measures most relevant to patients [1-6]. Patient-reported outcomes measures (PROMs) comprise data collected from individual patients and include an array of outcomes such as symptoms, daily functioning, and health-related quality of life (HRQoL). PROMs are increasingly used in daily clinical practice for individual patient management [7]. Individual PROMs data incorporates the patient's perspective on their health status and can detect issues that are most bothersome to the individual patient. By reporting these issues to both patients and clinicians, patient-physician communication improves [8-10]. This may support shared-decision making, and therefore offers considerable potential to enhance quality of care and clinical outcomes $[8,11]$. A second application of PROMs data feedback is the use of aggregated PROMs scores collected in clinical studies or trials to inform patients about treatment harms and benefits [12].

Currently, when PROMs are incorporated within clinical practice, raw or summarized PROMs data are given as feedback to patients and/or clinicians by using different graphic visualization formats [13]. In order for them to understand and apply the information during clinical encounters, patients and clinicians ought to interpret such formats correctly [13]. A previous review by Bantug et al. [13] reported that the majority of patients and clinicians were able to interpret plain or straightforward graphs. Bantug et al. suggested that future research should focus on optimizing graphic visualization strategies. After the publication of this review in 2016, considerable new evidence has become available about this topic. Moreover, the focus of recent studies has shifted towards the effect of aspects such as score directionality [14-17] and axis labelling $[1,15]$ on correct interpretation by patients and clinicians. Furthermore, there is increased attention for guiding the clinical interpretation of PROMs data, e.g. to distinguish severe or clinically relevant symptoms $[1,3-5,14,18]$. For instance, the display of score thresholds and warnings if scores change over time would be helpful in daily practice [1], as well as scores from reference populations to compare individual scores to $[1,5,14]$. Both facilitate the correct use of scores during clinical encounters. The focus on clinical interpretation led to the introduction of funnel plots $[2,19]$, heat maps [4], and icon arrays [3, $15,18,20]$, underlining the relevance of assessing a wider variety of graphic visualization formats.

While worldwide implementation of PROMs data collection in clinical practice keeps progressing [21], new evidence on graphic visualization formats for PROMs scores for interpretation by patients and clinicians has become available. This systematic literature review aims to (1) address the latest evidence for graphic visualization formats of PROMs in clinical practice, by extracting preferences and interpretation accuracy for patient and clinicians, and (2) investigate how clinically relevant PROMs scores are distinguished, in order to guide clinical interpretation of PROMs scores for their use during clinical encounters.

\section{Methods}

A systematic literature review was conducted according to the Preferred Reporting Items for Systematic Reviews and Meta-Analysis (PRISMA) guidelines [22].

\section{Search strategy}

An exhaustive search strategy was developed by a medical librarian (E.W.) experienced in systematic literature searches. The following search terms were included: ("Patient reported outcome (PRO)" OR "Health-Related 
Quality of Life") AND "data presentation/display" AND "health communication" AND ("cancer" OR "clinical decision-making"). We focused the search on oncology as we work in an oncological setting. Also, most of these studies have been conducted in oncology, as confirmed by our search and previously shown by Bantug et al. [13]. Literature from January 2000 to July 2020 was searched in MEDLINE (accessed through PubMed), Embase (assessed through Ovid Platform), PsycINFO (assessed through Ovid Platform), and CINAHL. The full search strategy is included in Additional file 1: Table 1. Duplicates were removed using the Bramer method [23]. A forward and backward reference check was performed on all final included articles.

\section{Review procedure}

After removing duplicates, two researchers (E.A., I.F.) independently reviewed potential abstracts. The researchers eliminated articles according to the predefined inclusion and exclusion criteria (see below). In case of discrepancies, a third researcher (K.d.L.) was consulted. Subsequently, both researchers (E.A., I.F.) independently reviewed the full text version of each paper for inclusion in the final selection. Disagreements were discussed between the three researchers (E.A., I.F., and K.d.L.) to reach consensus.

Studies were included when concerning patients of 18 years and older treated in clinical practice; addressed communication of individual level or group level PROMs data, using graphic visualisation formats, to either patients or clinicians; were published in English. Exclusion criteria were: studies without English or full-text version; systematic literature reviews; Delphi studies; studies where PROMs had not been completed by patients or were not applied or visually presented to patients and/or clinicians.

\section{Analyses}

Two researchers (E.A., K.d.L.) independently extracted data from the included articles. The findings of both researchers were compared and verified by a third researcher (I.F.). First, study characteristics were described, including study goal, population, and type of PROMs data that was visualised. Second, findings about visualisation preferences and interpretation accuracy were extracted. Furthermore, methods and strategies for guiding the interpretation of scores during clinical encounters were extracted. The findings were described for patients and clinicians separately, and a distinction was made between individual PROMs data for use during clinical encounters, and mean or aggregated PROMs data that could be included in treatment decision-making.

\section{Quality assessment}

Two researchers (E.A., K.d.L.) independently assessed the methodological quality of the papers and compared their final judgments. The Critical Appraisal Skill Program (CASP) was used for methodological assessment of the included papers. CASP enables to systematically assess the trustworthiness, relevance, and results of published papers by comprising several criteria for qualitative studies, randomized controlled trials, and cohort studies [24]. The reviewers scored the papers per criteria with a positive or negative response, or 'not applicable/ unknown'. Studies with a positive score for half or more of the criteria were deemed of sufficient quality [24].

\section{Results}

Our search retrieved 1673 studies, from which 414 duplicates were excluded. Subsequently, from the remaining 1259 studies, 1186 were excluded; these did not describe PROMs data visualization (Fig. 1). Then, full-text articles from 73 eligible studies were assessed. From these, 47 were excluded based on inclusion and exclusion criteria, and/or because no full-text version was available $(n=15$, all conference abstracts). Ultimately, 25 studies were included in this review for data extraction (Additional file 1: Table 2). After quality assessment following the CASP criteria, all studies had a positive score for half or more of the criteria (Additional file 1: Table 3).

Most studies used either mixed methods design $(\mathrm{n}=12)$, including human-centered design, or a qualitative design $(n=9)$, including interviews (Fig. 2). Sample sizes ranged from 8 (interview study) to 1,017 (survey study). Studies had been carried out in different clinical domains and in different countries, studying different graphic visualization formats and designs. The majority of studies included participants during or after treatment, whereas nine studies made use of hypothetical settings. PROMs data formats were either based on individual patient scores presented to patients $(\mathrm{n}=17)$ and/or clinicians $(\mathrm{n}=14)$, or based on mean group-reported data from for instance clinical trials that were presented to patients $(\mathrm{n}=10)$ and clinicians $(n=8)$. The different graphic visualization formats that were studied are presented in Fig. 3. The results are presented according to the distinction between patients and clinicians, subdivided into preferences and interpretation accuracy.

\section{Individual level PROMs data-patients}

An overview of the extracted data for patients on individual and group level is presented in Table 1. 

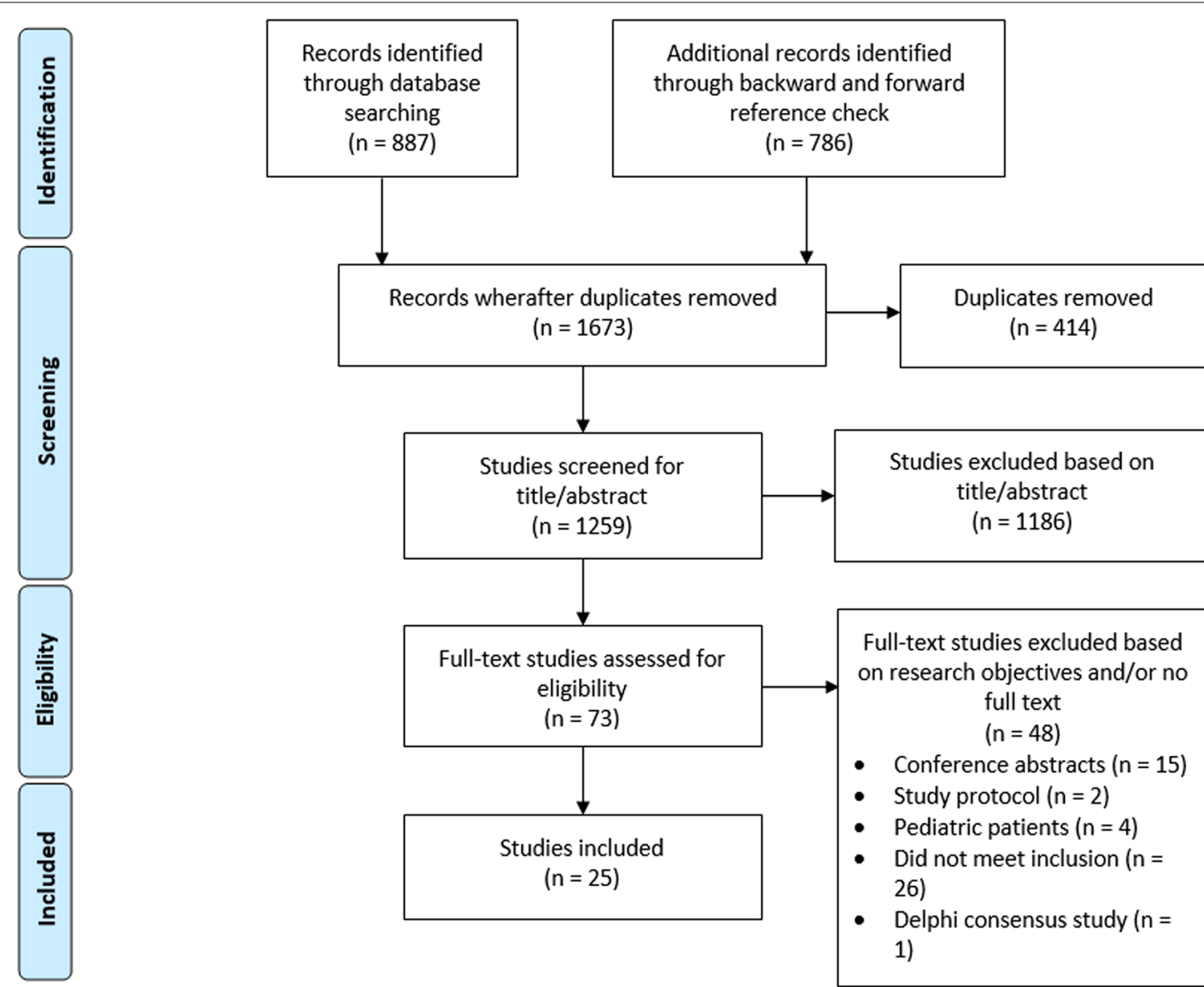

Fig. 1 PRISMA flow chart of literature search and review process

\section{Preferences}

From the 25 studies included in this study, 12 assessed patients' preferences. Patients appreciated that their individual PROMs scores were presented visually over time [16, 25]. Six studies reported that patients preferred bar charts over other graphic visualization formats for reporting longitudinal PROMs data [1, $3-6,14]$. Bar charts were 'visually clear', and facilitated 'appraisal at a glance', since bar charts are a generally well-known format for presenting data [2]. Five studies reported that line graphs were preferred as well [1, $6,15,16,25]$. Patients experienced line graphs as 'easy to understand' and 'quick to retrieve relevant information from' regarding their symptoms and well-being [1]. Furthermore, line graphs were preferred for overall ease-of-understanding and usefulness [16].

\section{Interpretation accuracy}

Eight studies assessed patients' interpretation accuracy for visualizing individual PROMs data. Bar charts were more often interpreted correctly $(87.8 \%)$ compared to line graphs (74.3\%) [14]. According to Geerards et al. [26], a study conducted among 1386 participants of the general population, $92.9 \%$ of participants thought that bar charts were clear, and $82.4 \%$ thought bar charts were accurate. In a study by Loth et al. [17], among 40 patients, 90\% reported that bar charts were "very easy" or "rather easy" to understand. Nevertheless, Grossman et al. [27] described that from 12 included patients, half of them failed to interpret bar charts correctly, and even patients who successfully read it, often required multiple attempts to do so. These participants were, however, introduced to bar charts for the first time [27]. One study reported that line graphs could be interpreted accurately by patients; nonetheless, an undefined proportion of patients needed initial guidance on how to read line graphs [1]. Liu et al. [28] reported that from 25 respondents (patients), several did not notice or understand the longitudinal nature of a line graph from left to right, particularly those with limited health literacy. Furthermore, a few patients misinterpreted a 


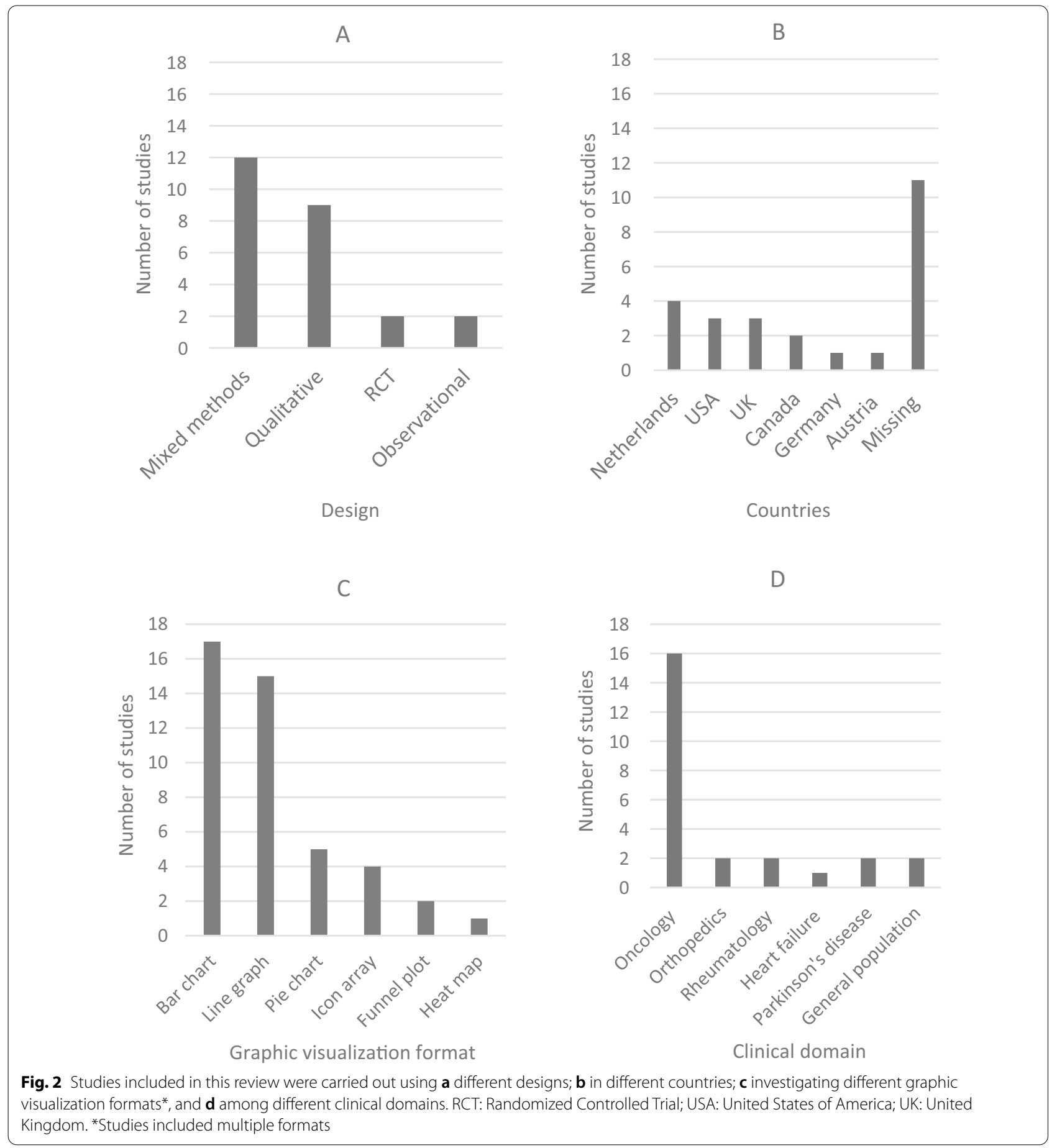

line drawn between two data points to mean information from between visits [28].

\section{Group-level or aggregated PROMs data-patients} The aim of presenting aggregated or mean PROMs scores to patients is to inform them about potential treatment outcomes, in order to support shared decision-making. In the included studies, aggregated PROMs data were mainly derived from clinical studies or RCTs. These were often presented to patients to (hypothetically) inform them about differences in outcomes between two treatments [12, 15, 20, 29-33]. 


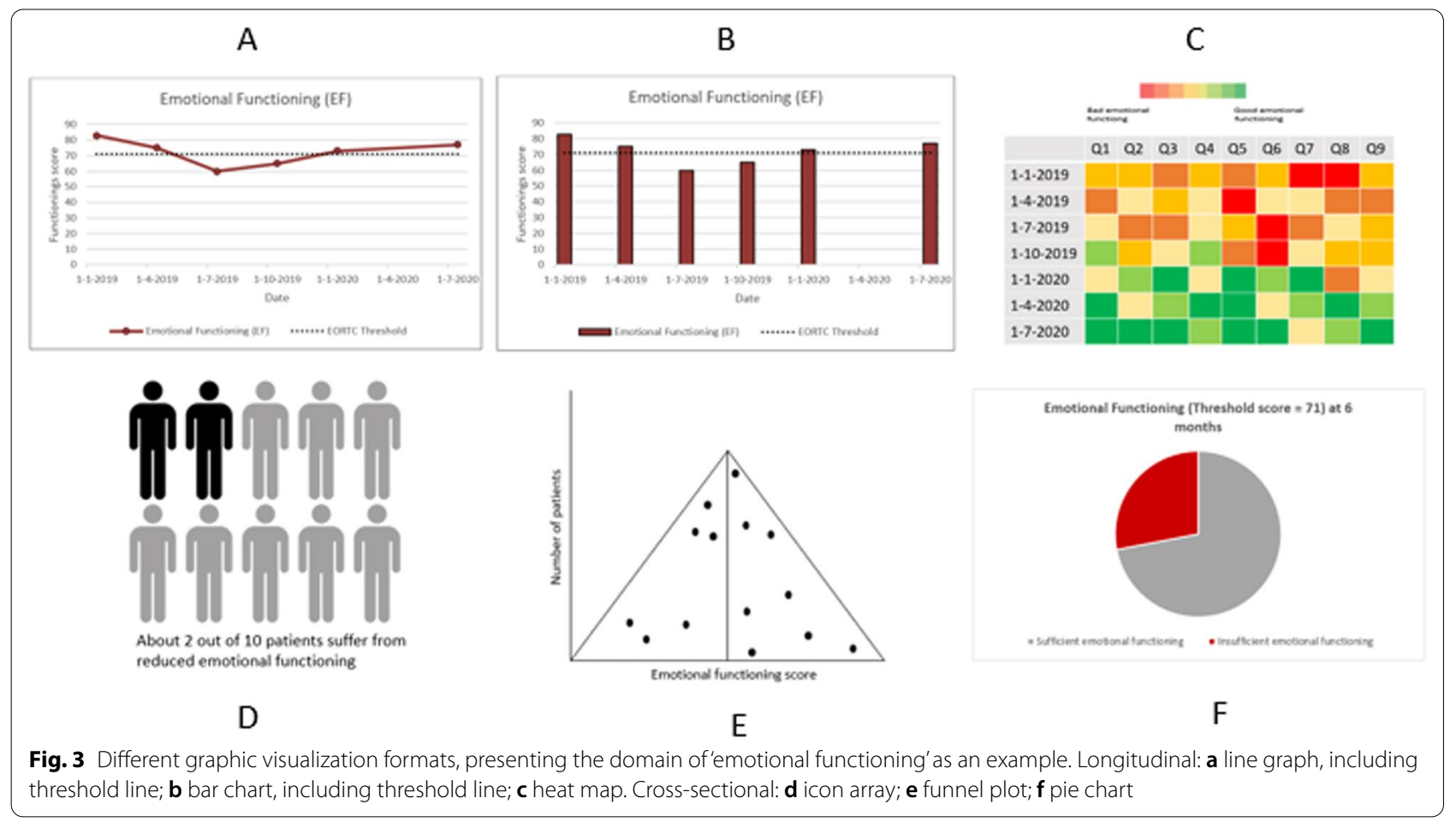

\section{Preferences}

Seven studies assessed patients' preferences for presenting aggregated outcomes over time. Four studies reported that patients preferred line graphs over bar and pie charts $[12,14,16,30]$. Line graphs were preferred because of their relative simplicity, straightforward layout, and perceived helpfulness $[12,16,30]$. The preference for line graphs decreased when confidence intervals were presented [12]. To present proportional data, three studies reported that patients preferred pie charts [1820]. Pie charts were easy to read and enabled obtaining information quickly [20]. Smith et al. [18] reported that of 39 patients, $55 \%$ preferred pie charts, compared to a $25 \%$ preference for icon arrays, and a $20 \%$ preference for bar charts. Van Overveld et al. [19] reported that among 37 patients, pie and bar charts were both preferred.

\section{Interpretation accuracy}

Seven studies assessed patients' interpretation accuracy for presenting aggregated outcomes over time. In four studies, patients' most often correctly interpreted line graphs $[14,16,29,30]$. Interpretation accuracy ranged from $98-100 \%$ [16, 30]. In particular, $56 \%$ of patients correctly answered questions for "better" (i.e. higher score means better outcomes) formatted lines, compared to $41 \%$ for "more" (i.e. higher score means more symptoms), and 39\% for "normed" (i.e. score as compared to reference scores) line graphs [29]. Line graphs showing results of two treatment options resulted in decisions that reflected adequate comprehension of the information in the graph [14]. One study reported that patients' accuracy was highest for pie charts and icon arrays, compared to bar charts [20]. Icon arrays were easy to understand for patients as well [20].

\section{Individual level PROMs data-clinicians}

An overview of the extracted data for clinicians on individual and group level is presented in Table 2.

\section{Preferences}

Thirteen studies assessed clinicians' preferences regarding visualization of PROMs scores from individual patients. In general, clinicians appreciated viewing PROMs scores repeated over time, in order to track their patients' symptom experiences $[14-16,25,35$, 36]. Moreover, six studies showed that bar charts were most preferred when plotting longitudinal individual PROMs data $[1,2,4,5,14,17]$. Furthermore, line graphs were preferred in four studies [1, 14-16, 29]. However, Brundage et al. [37] and Izard et al. [3] both stated that clinicians did not universally find one approach for longitudinal data as appealing or preferred. Contrastingly, a study among 227 health professionals by Kuijpers et al. [4] showed that the majority of medical specialists (46\%) and nurses (32\%) preferred heat maps to line graphs and bar charts. 


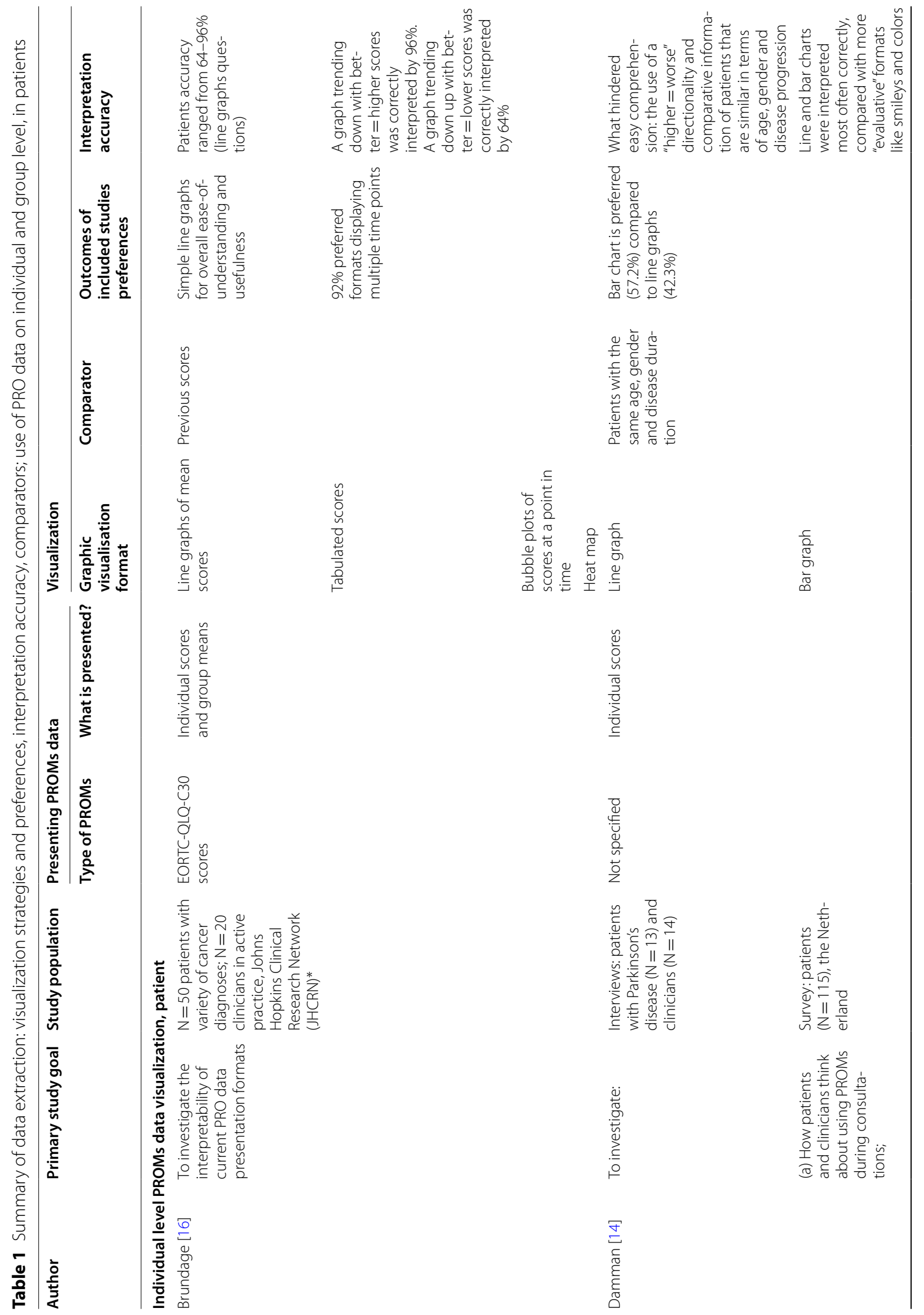




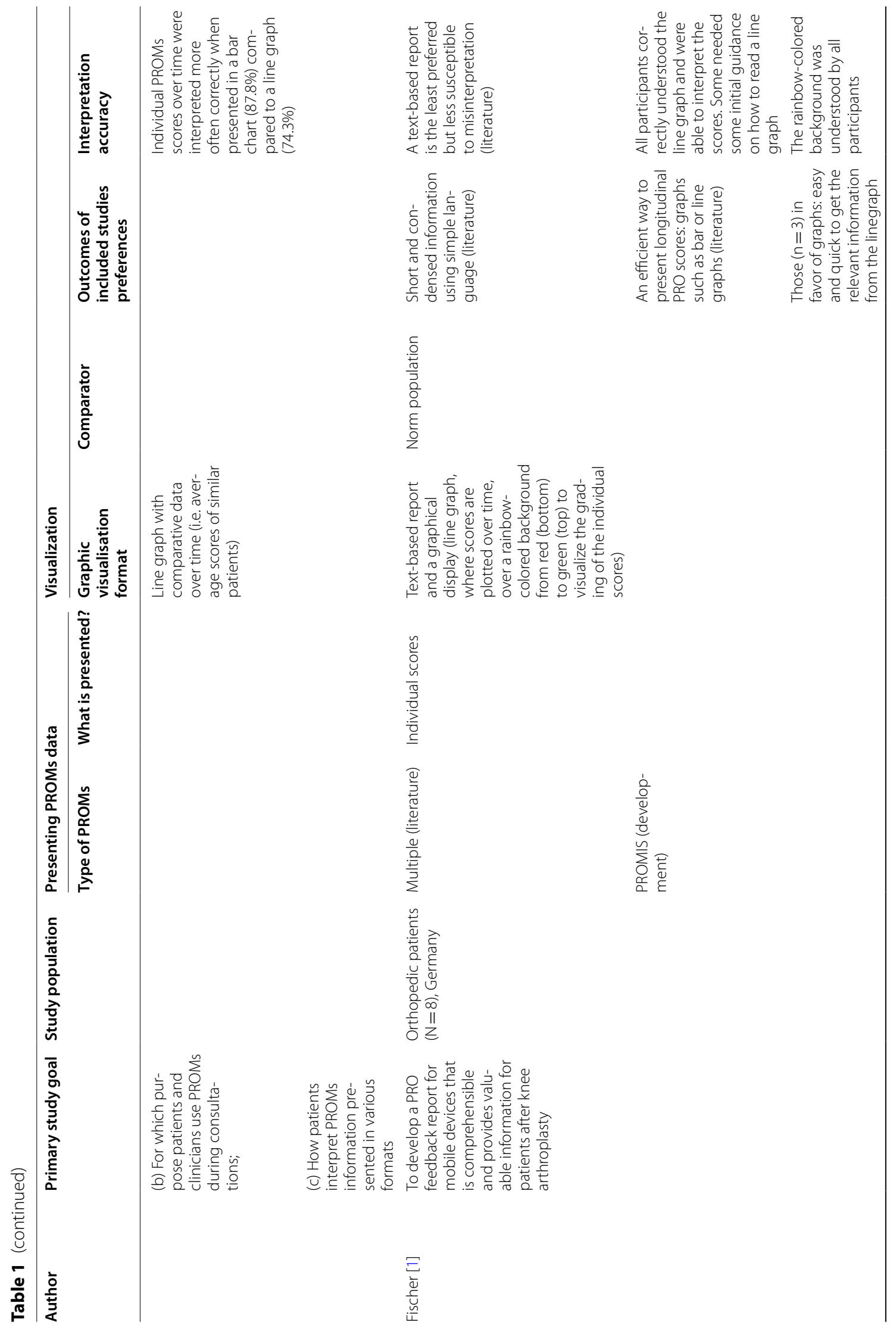




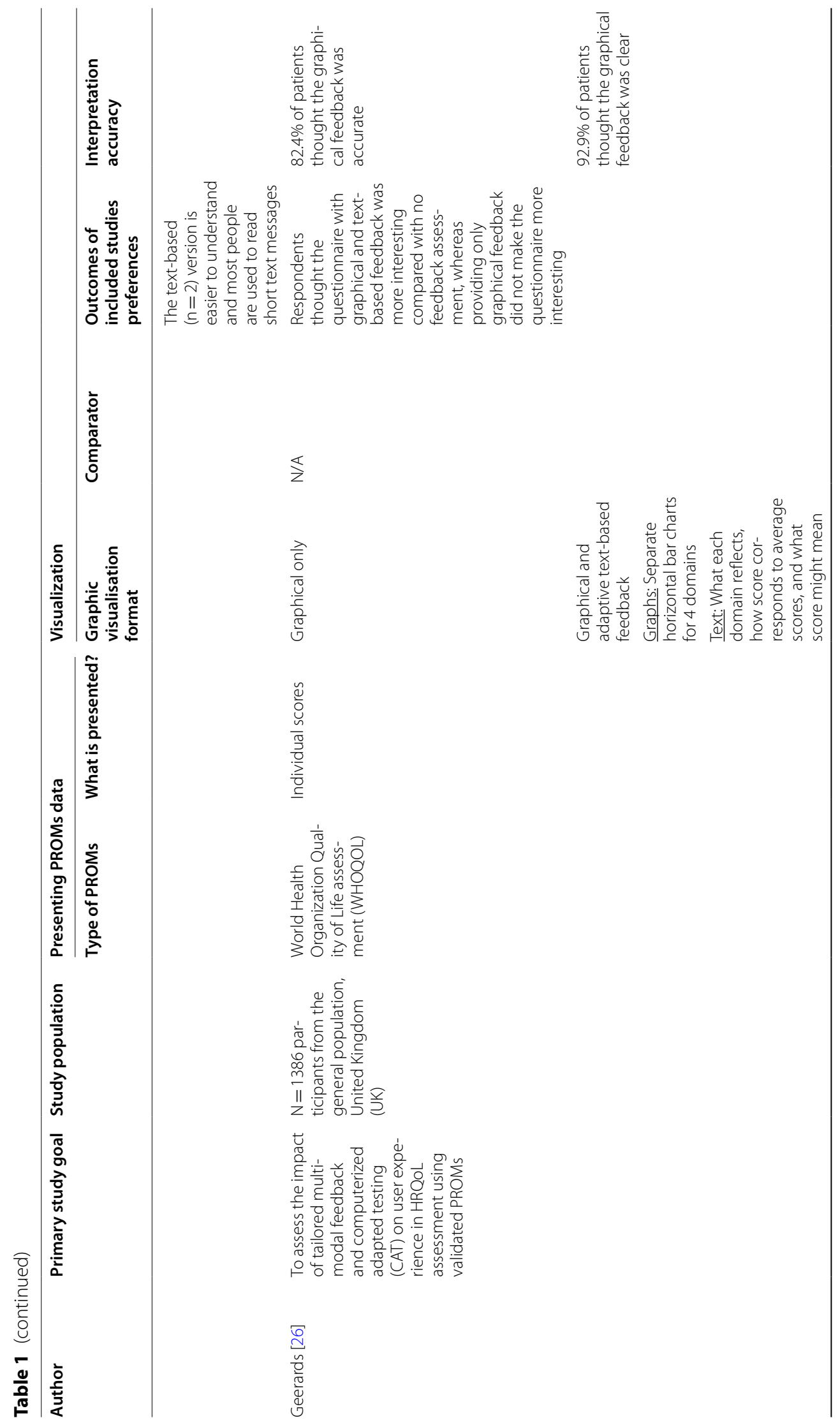




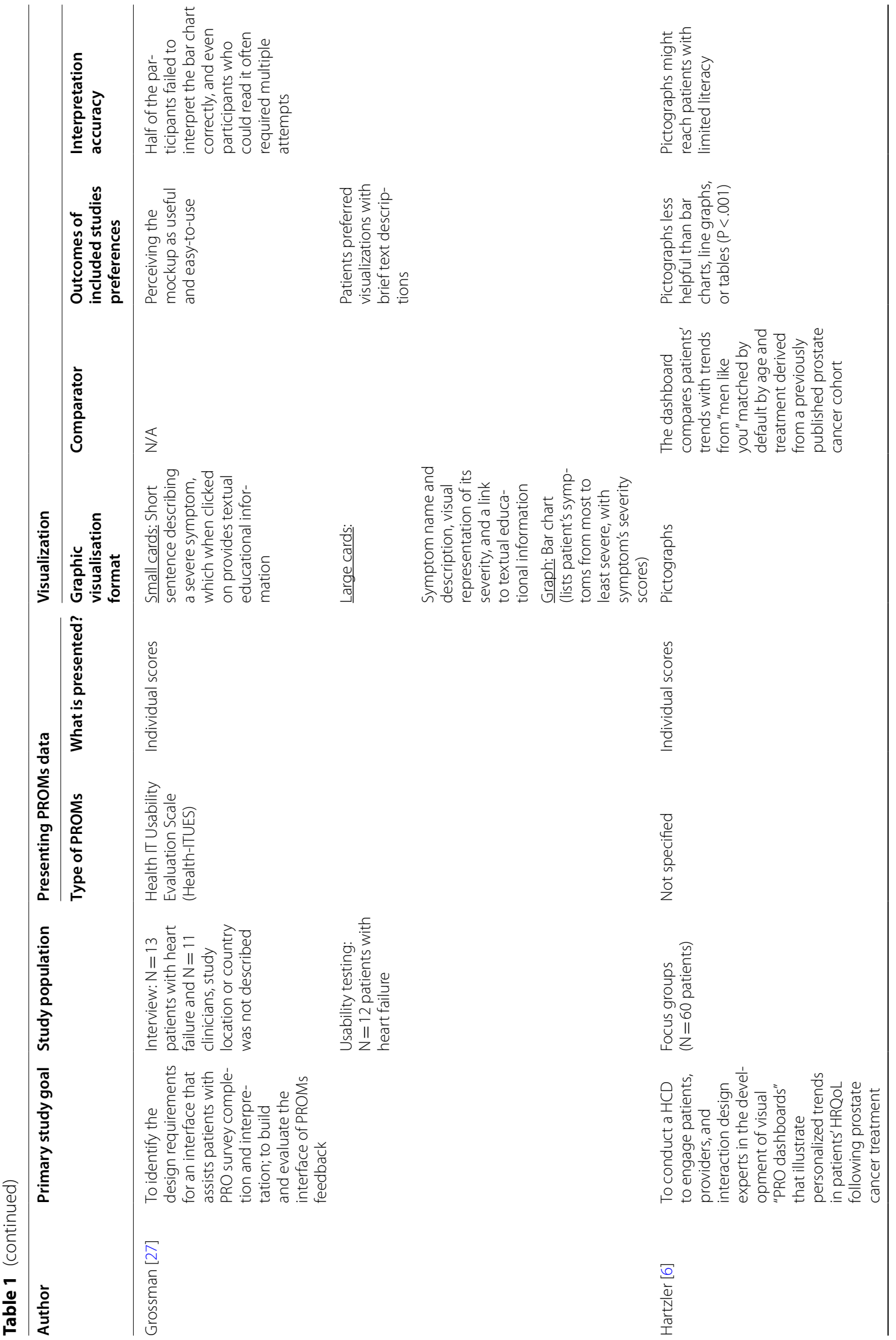




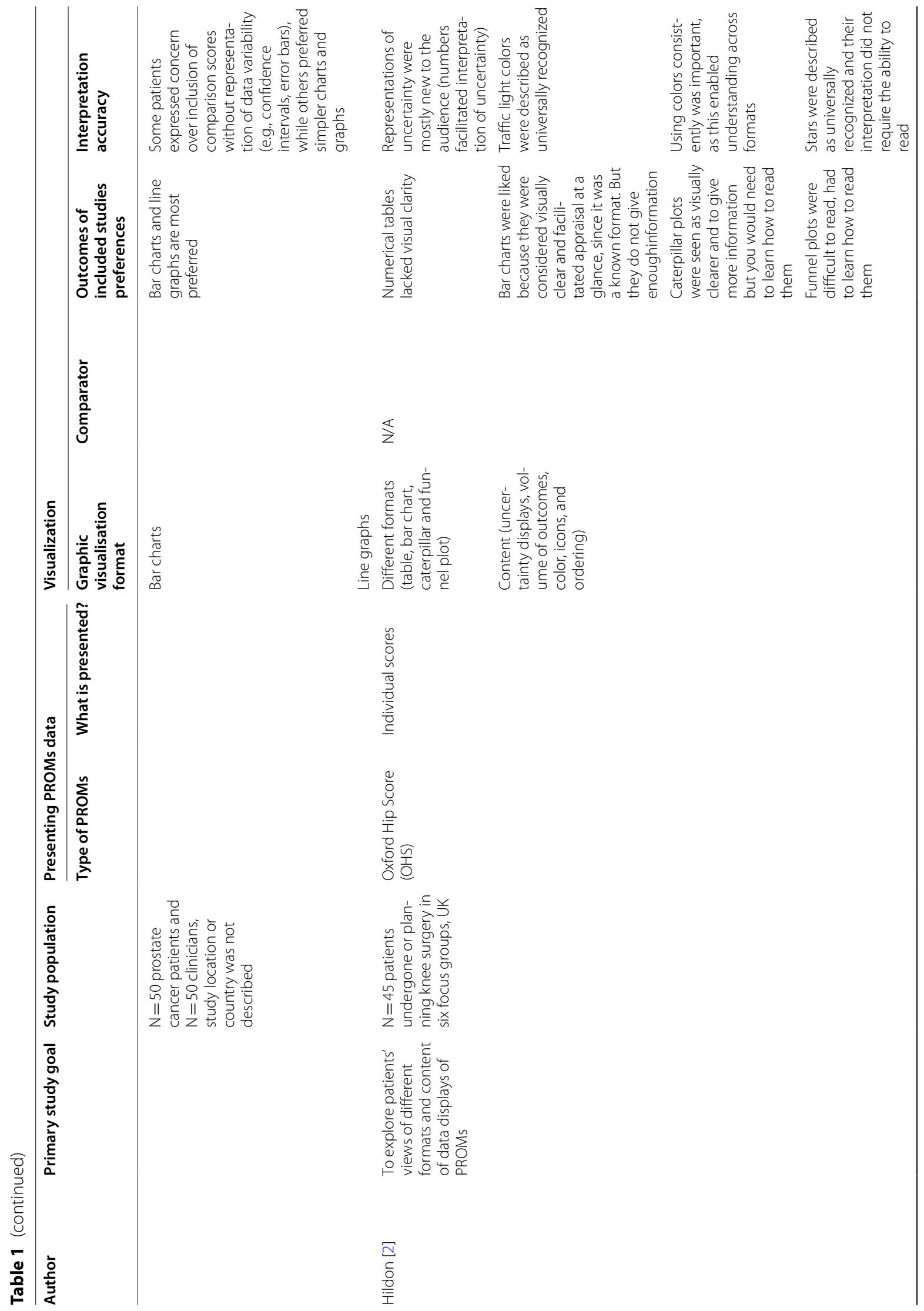




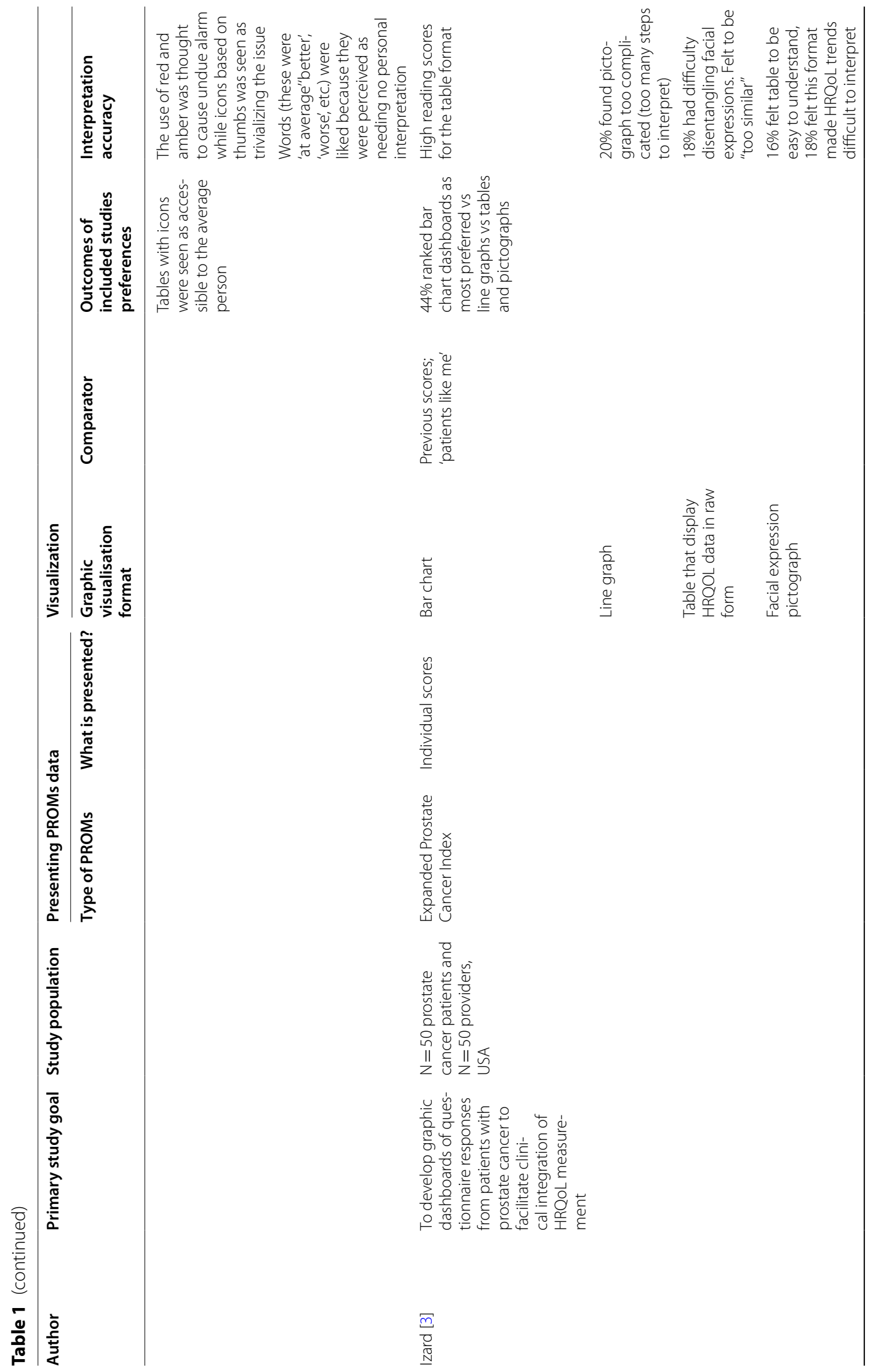



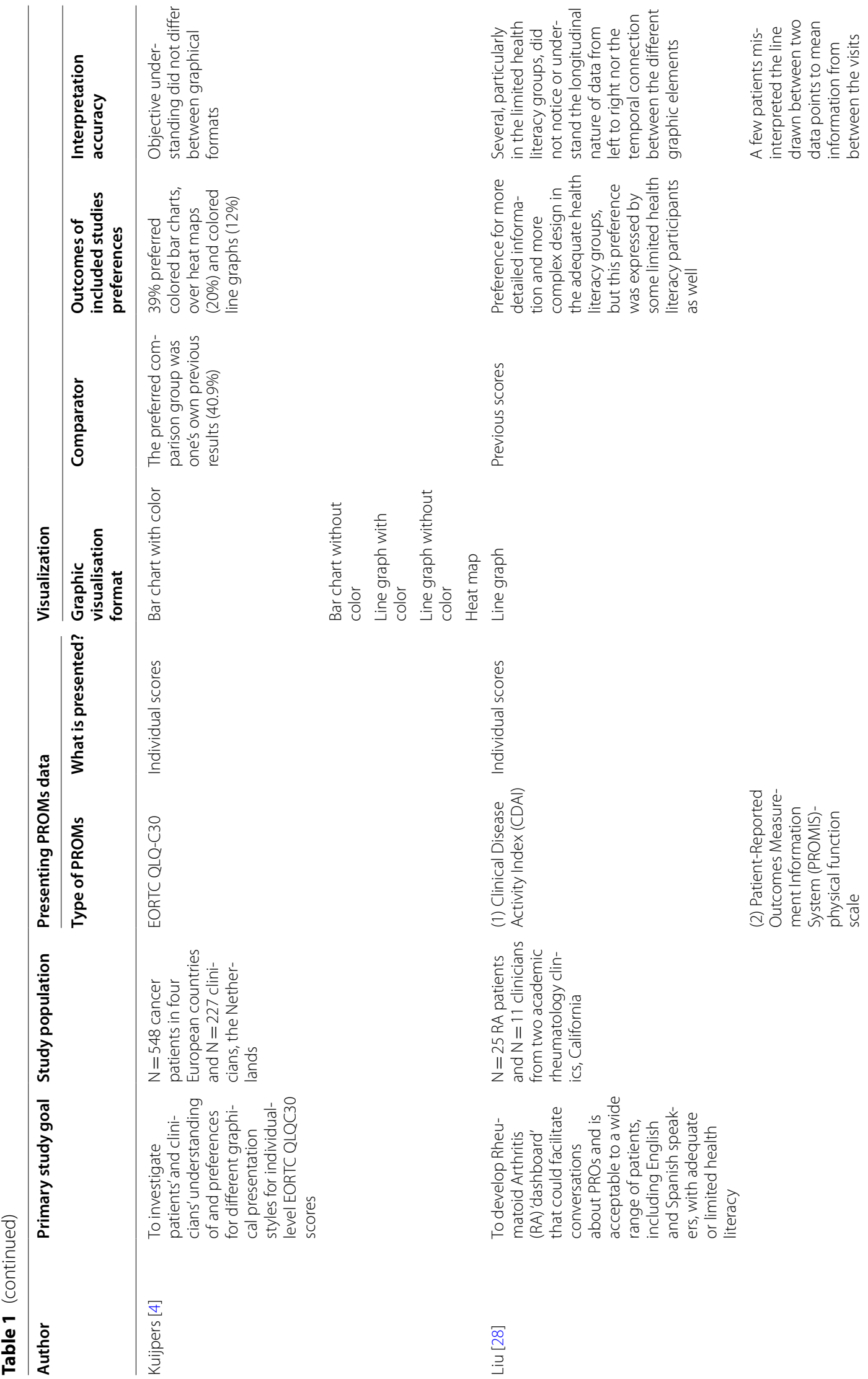

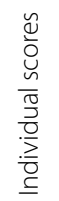
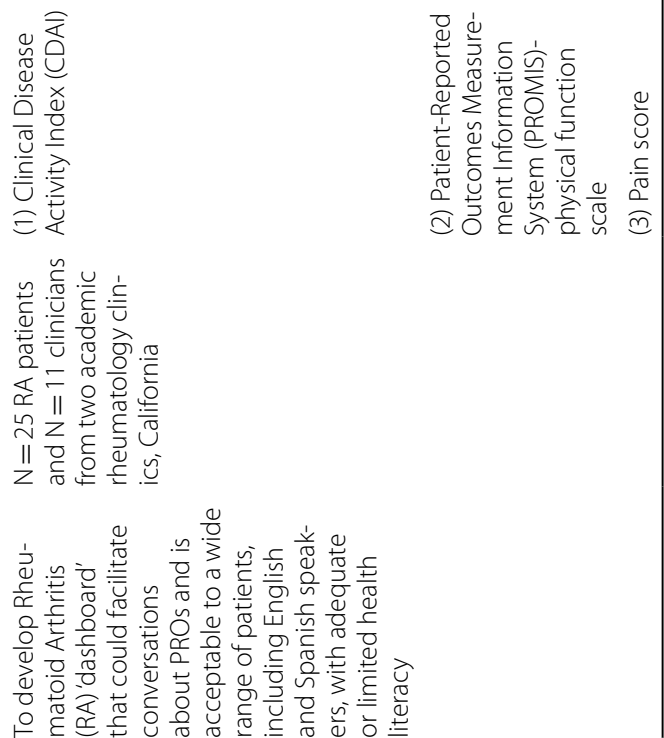

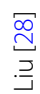




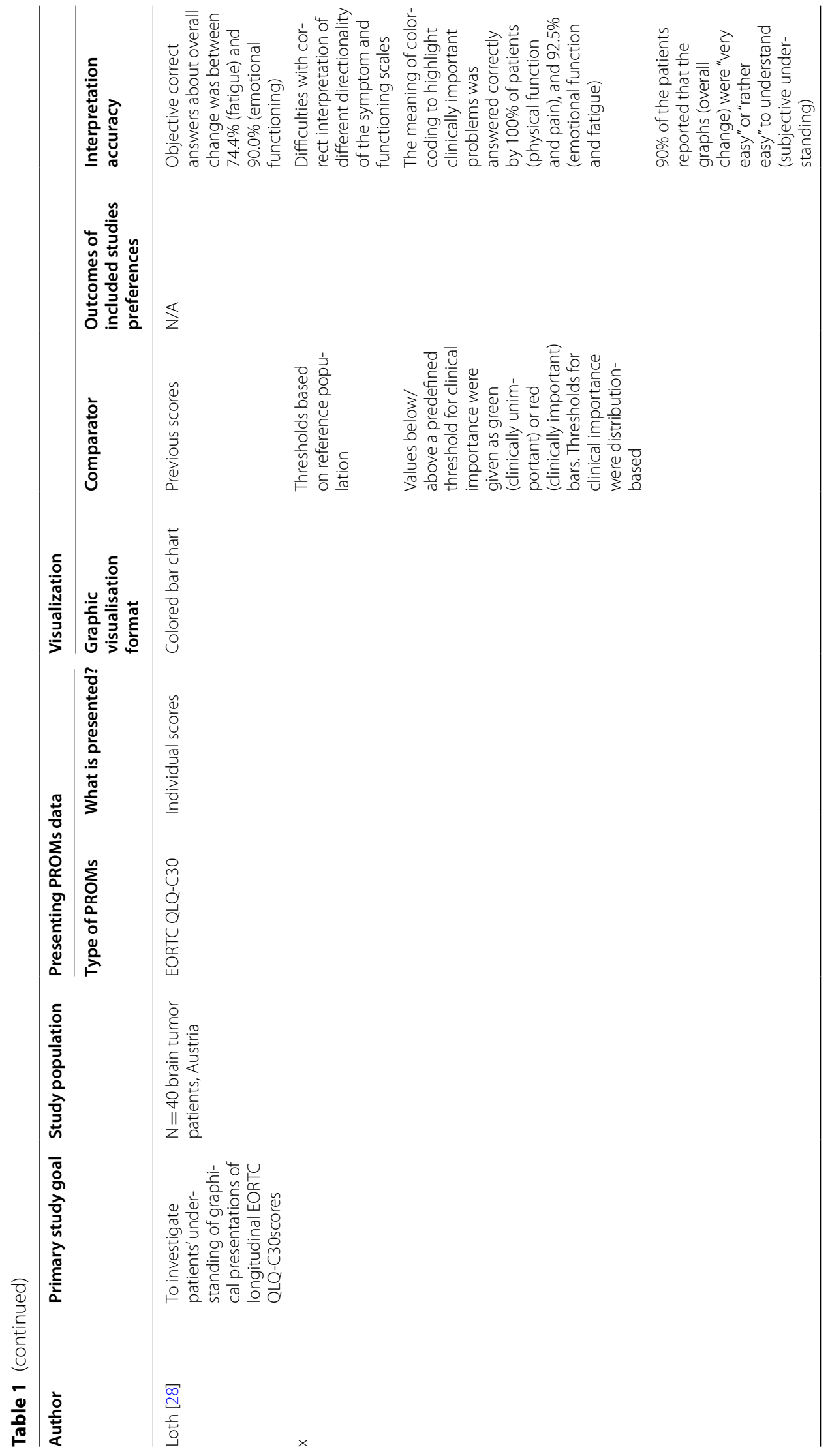




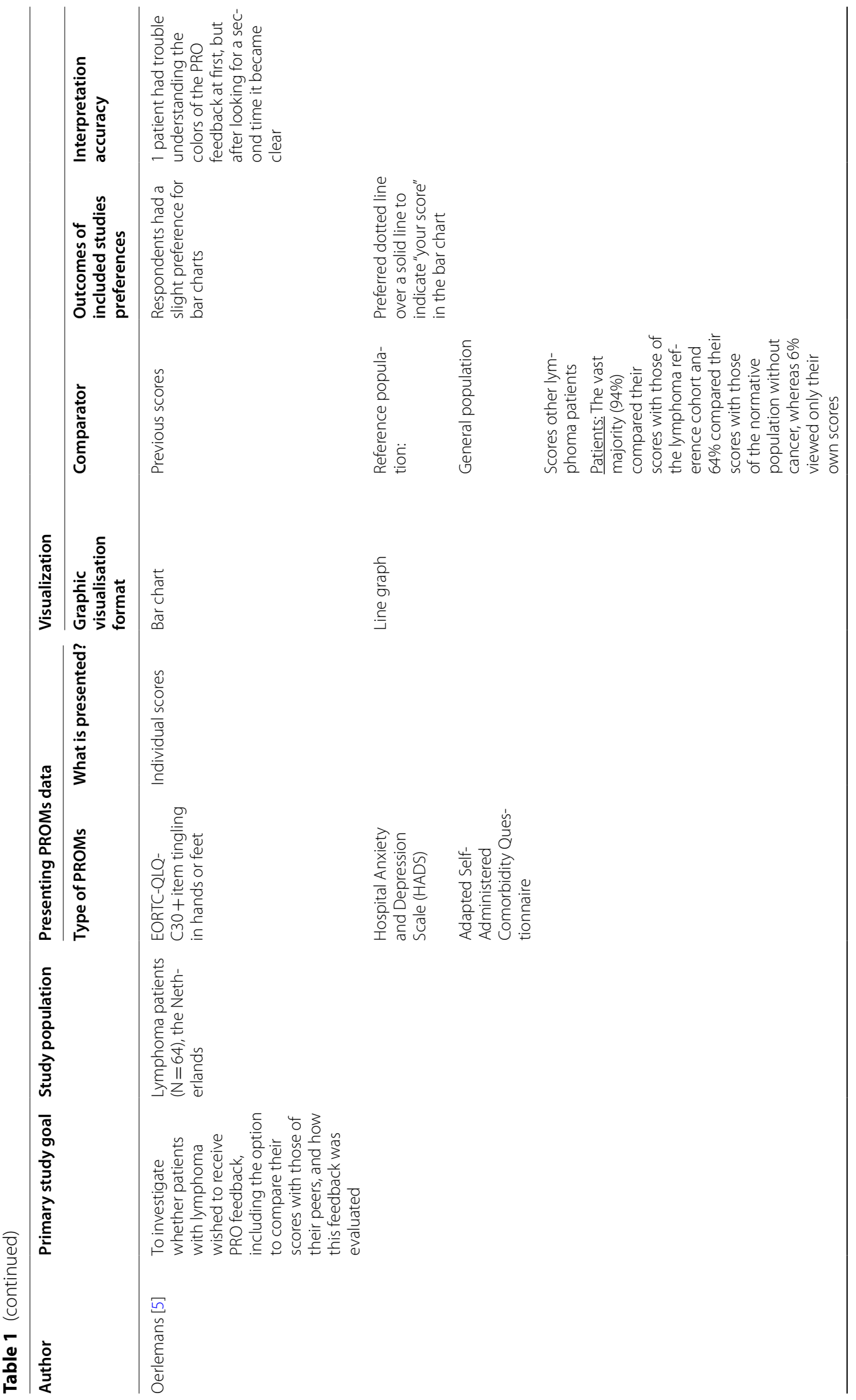




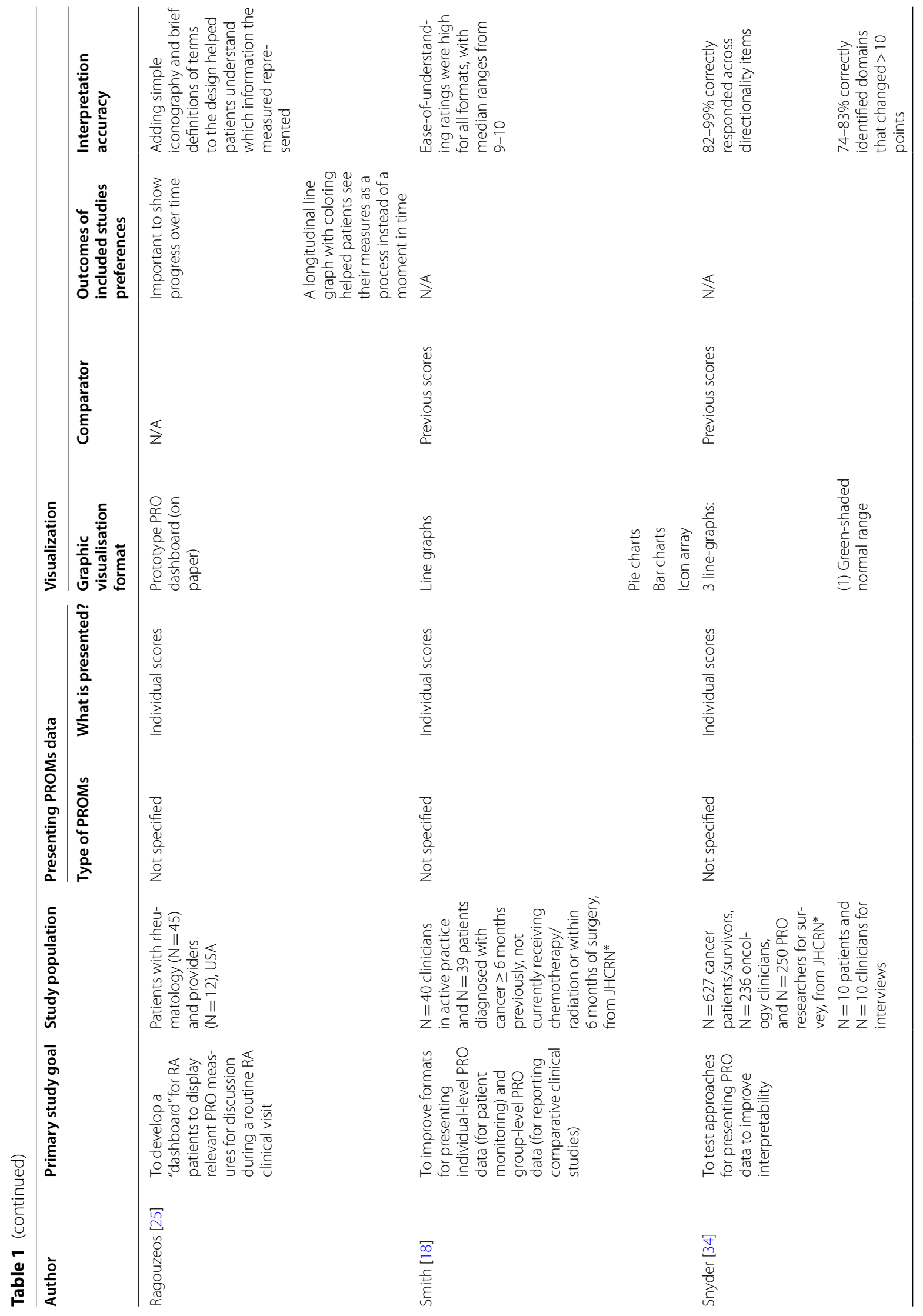




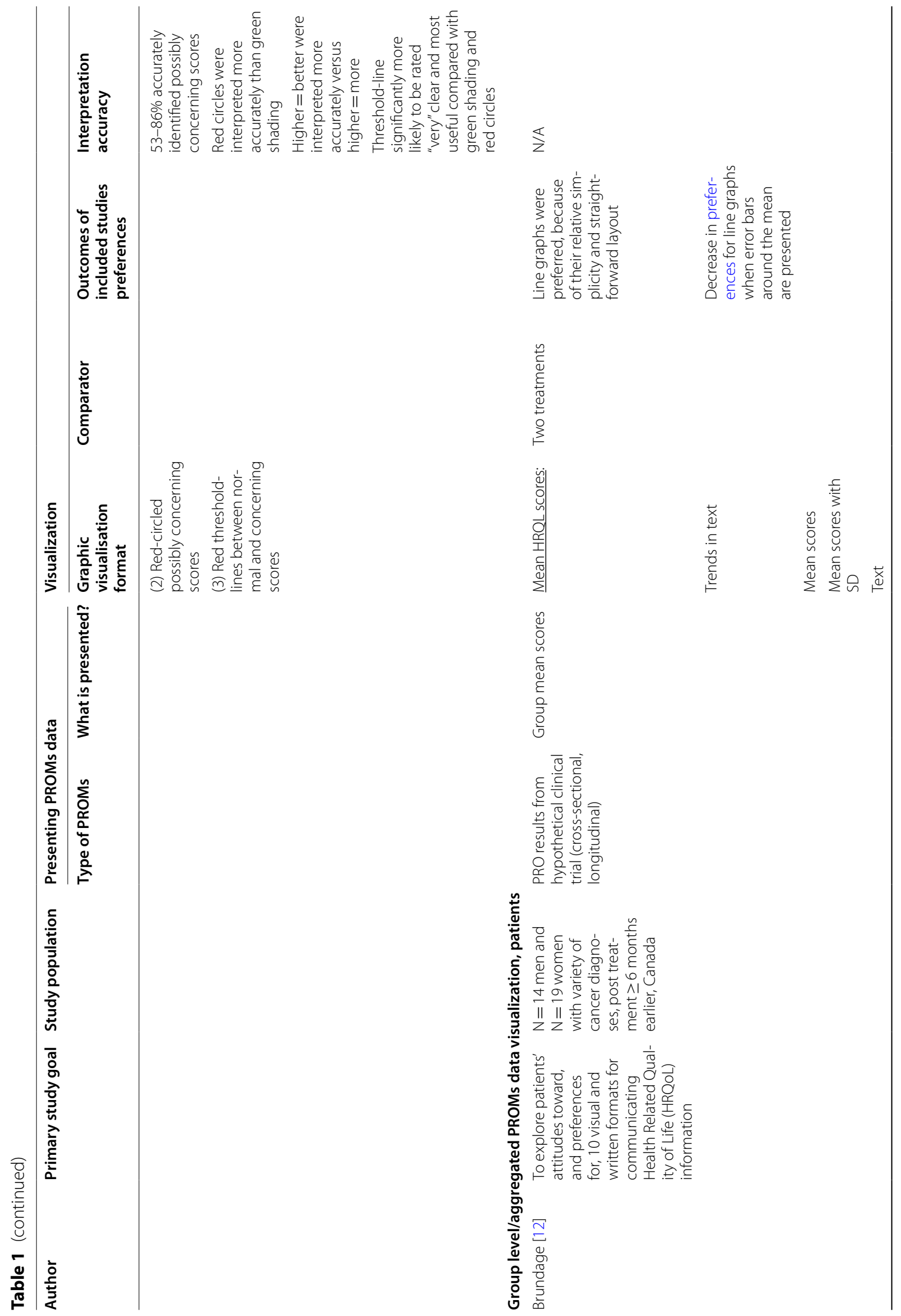




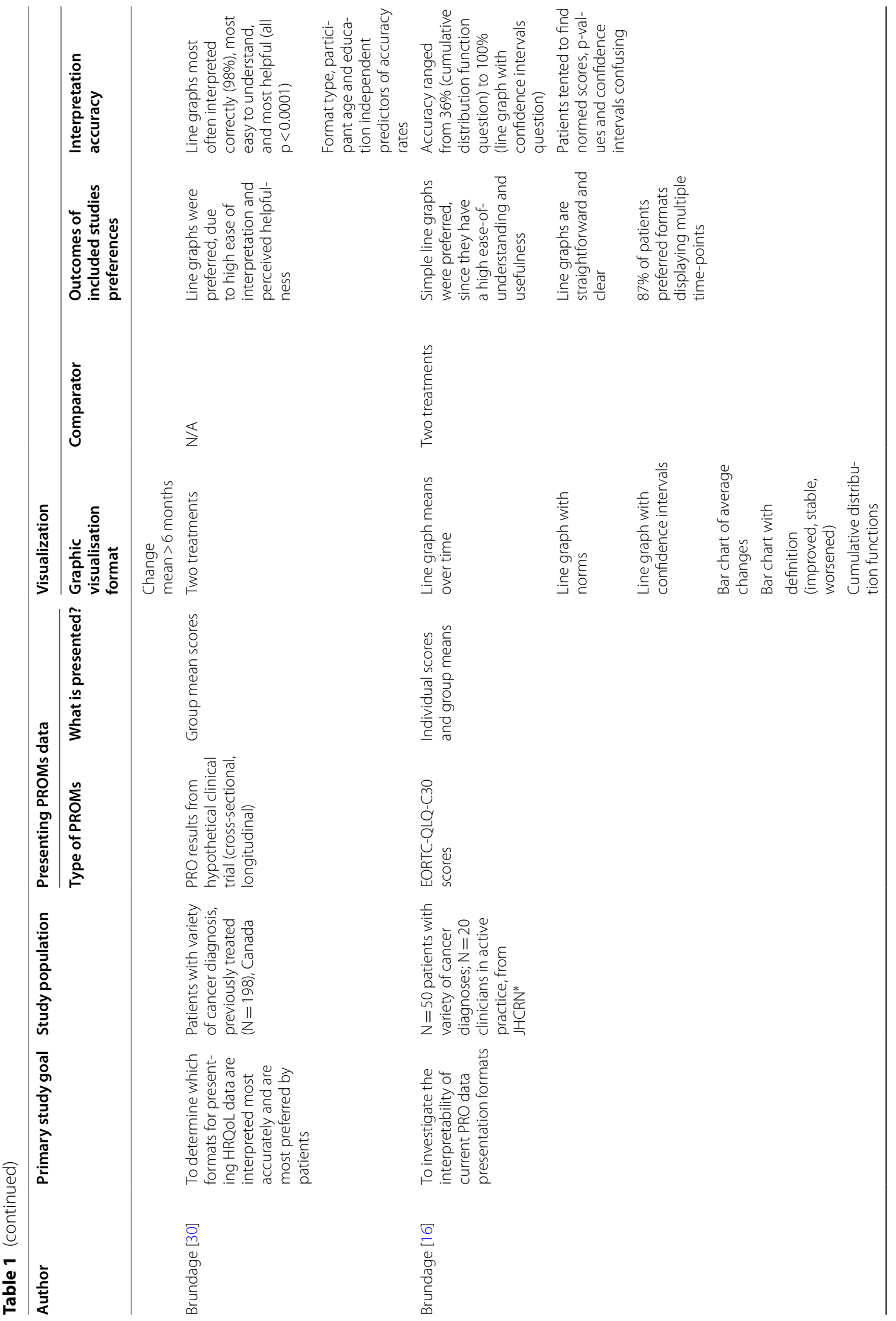




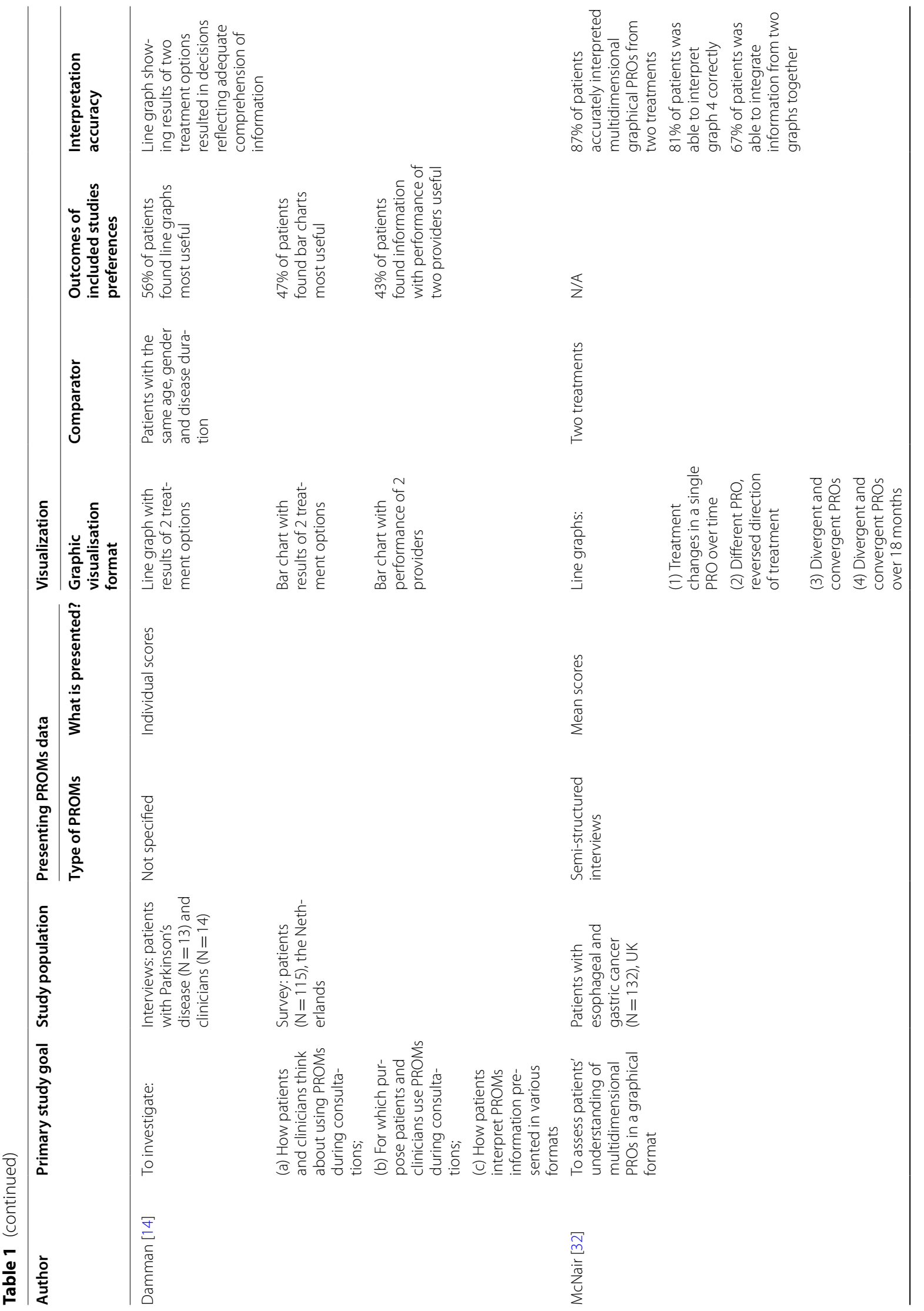




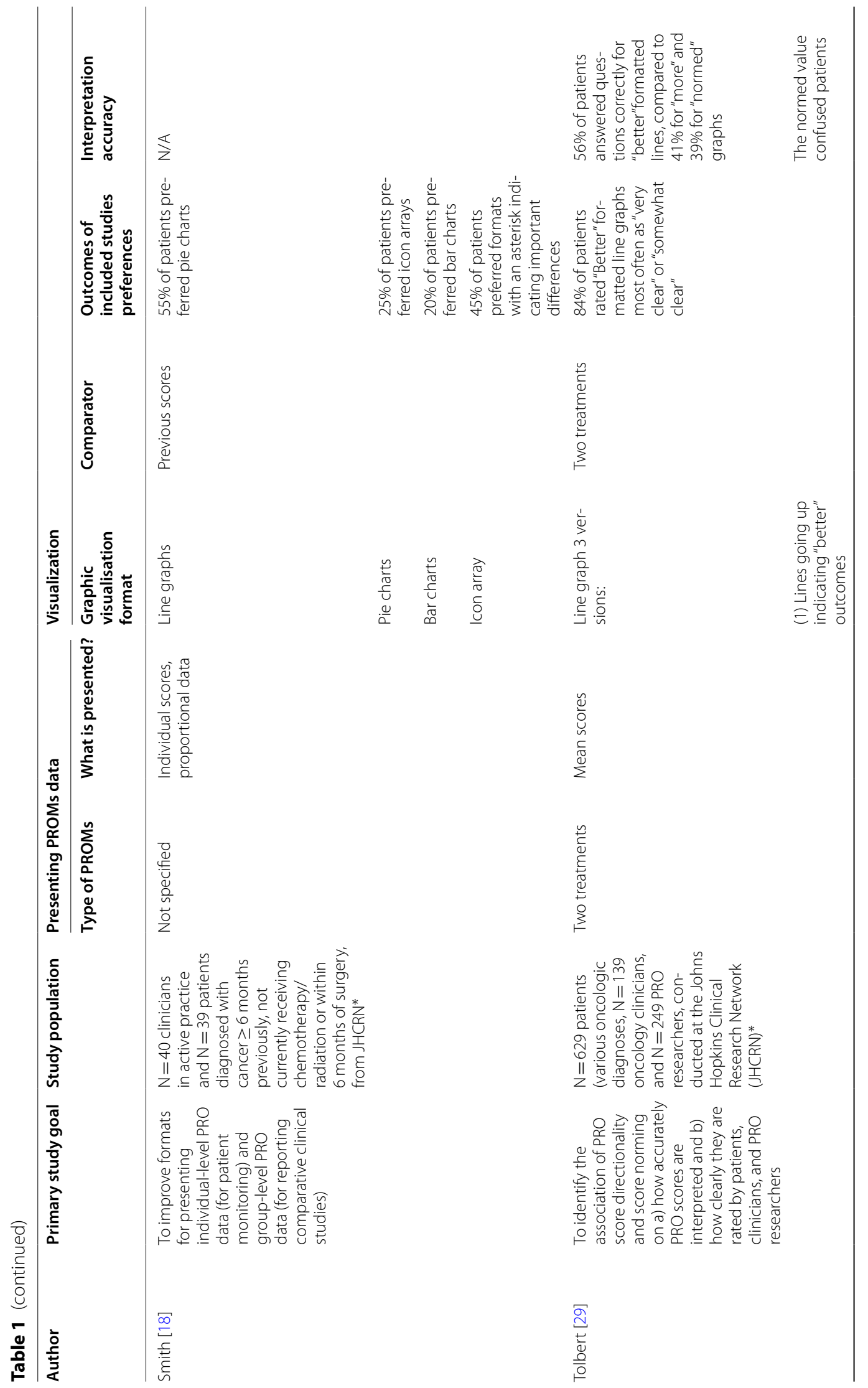




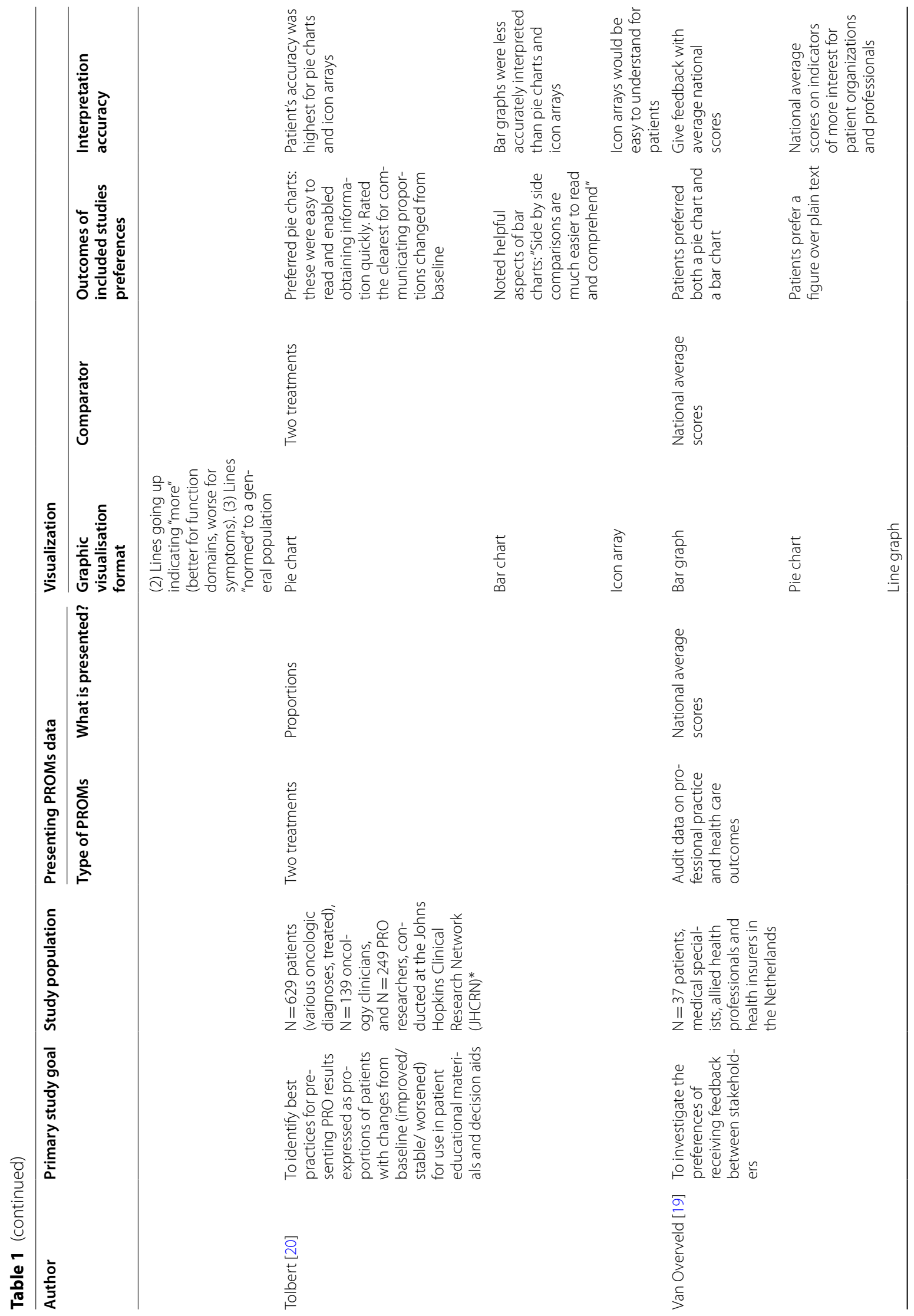




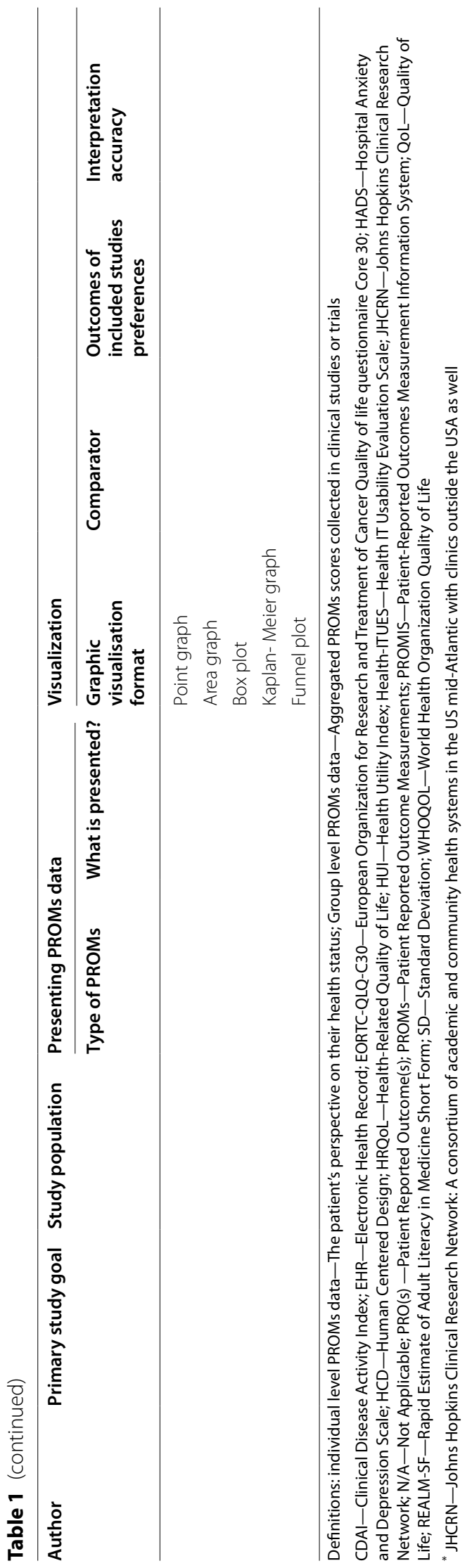




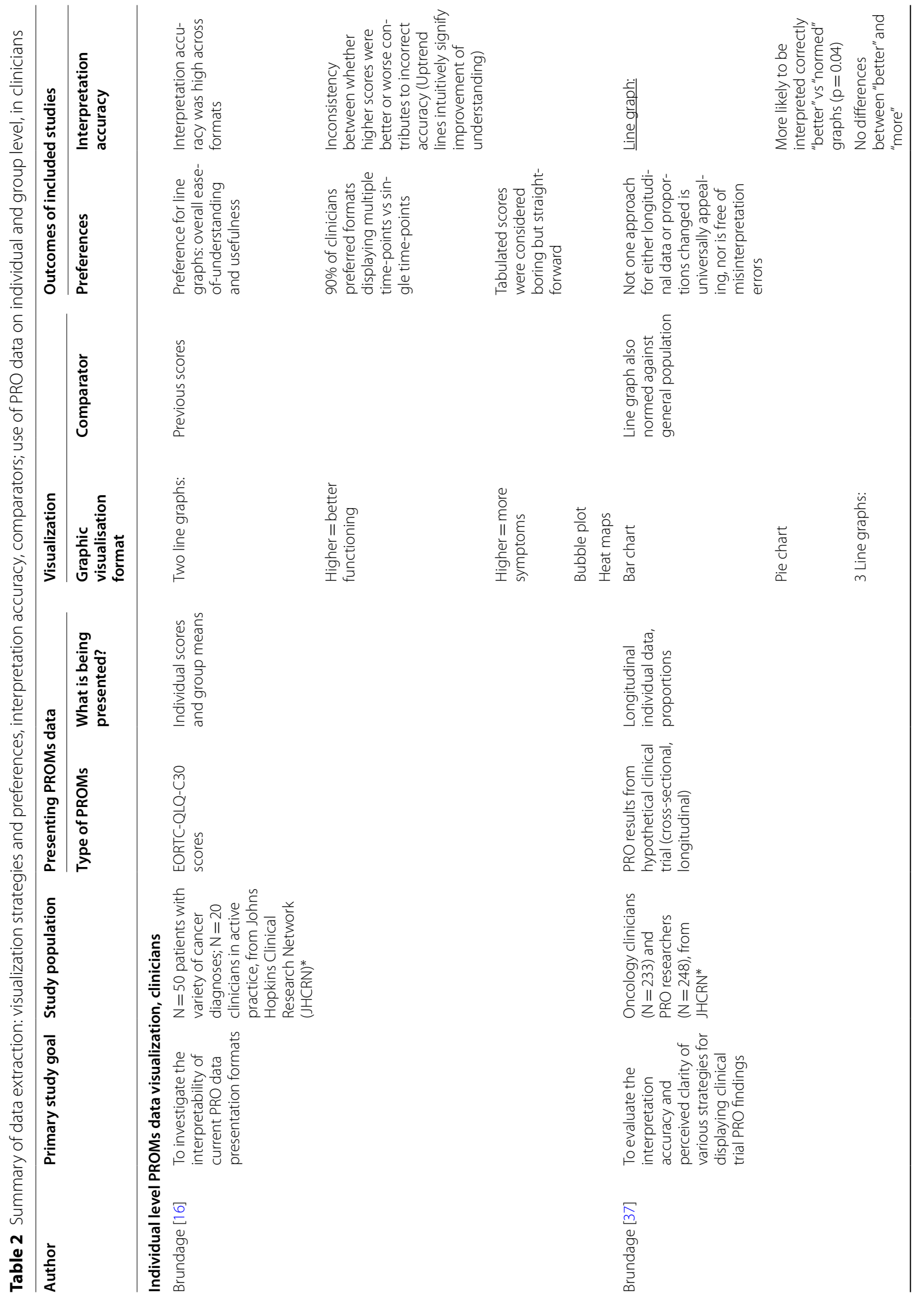




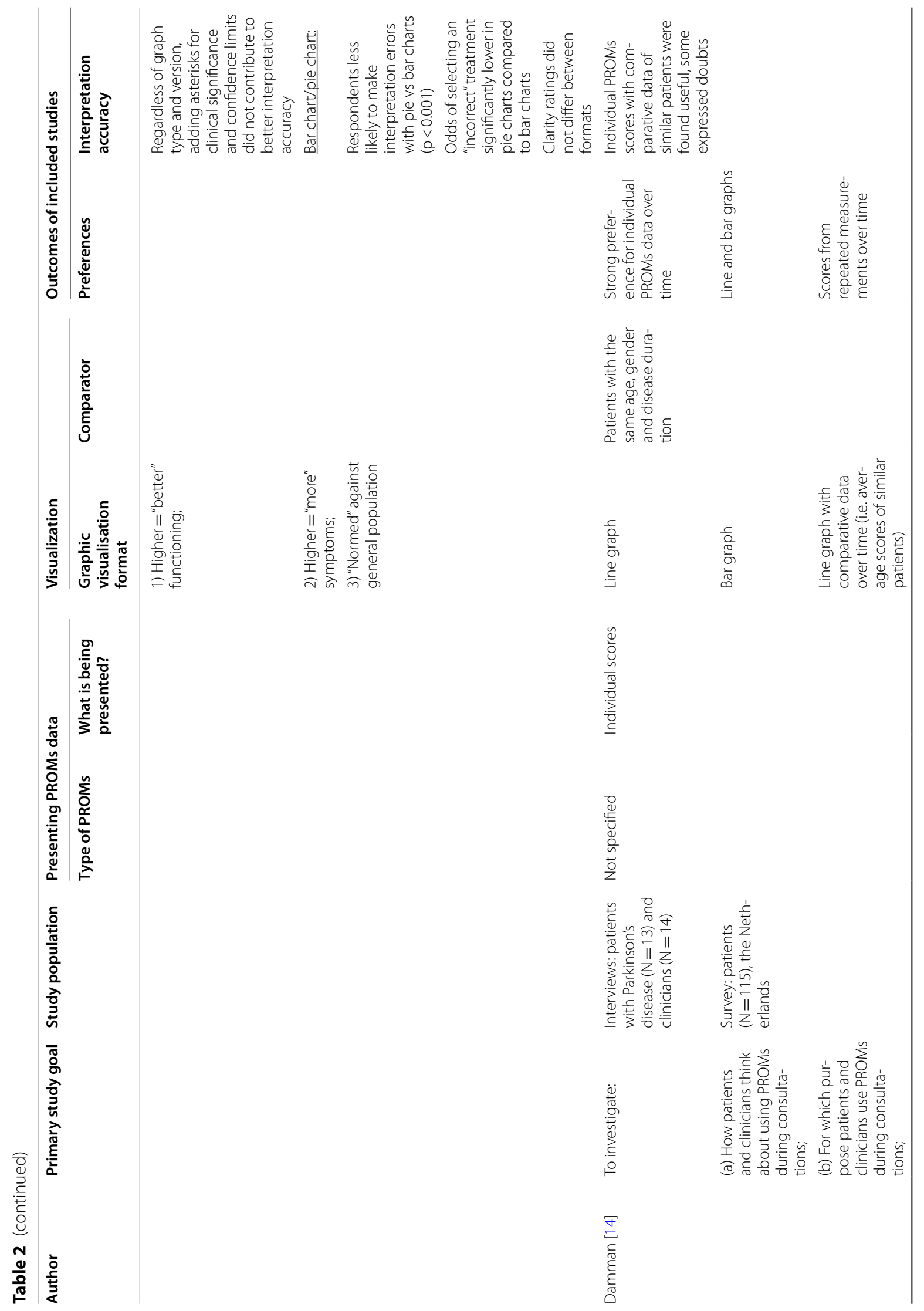




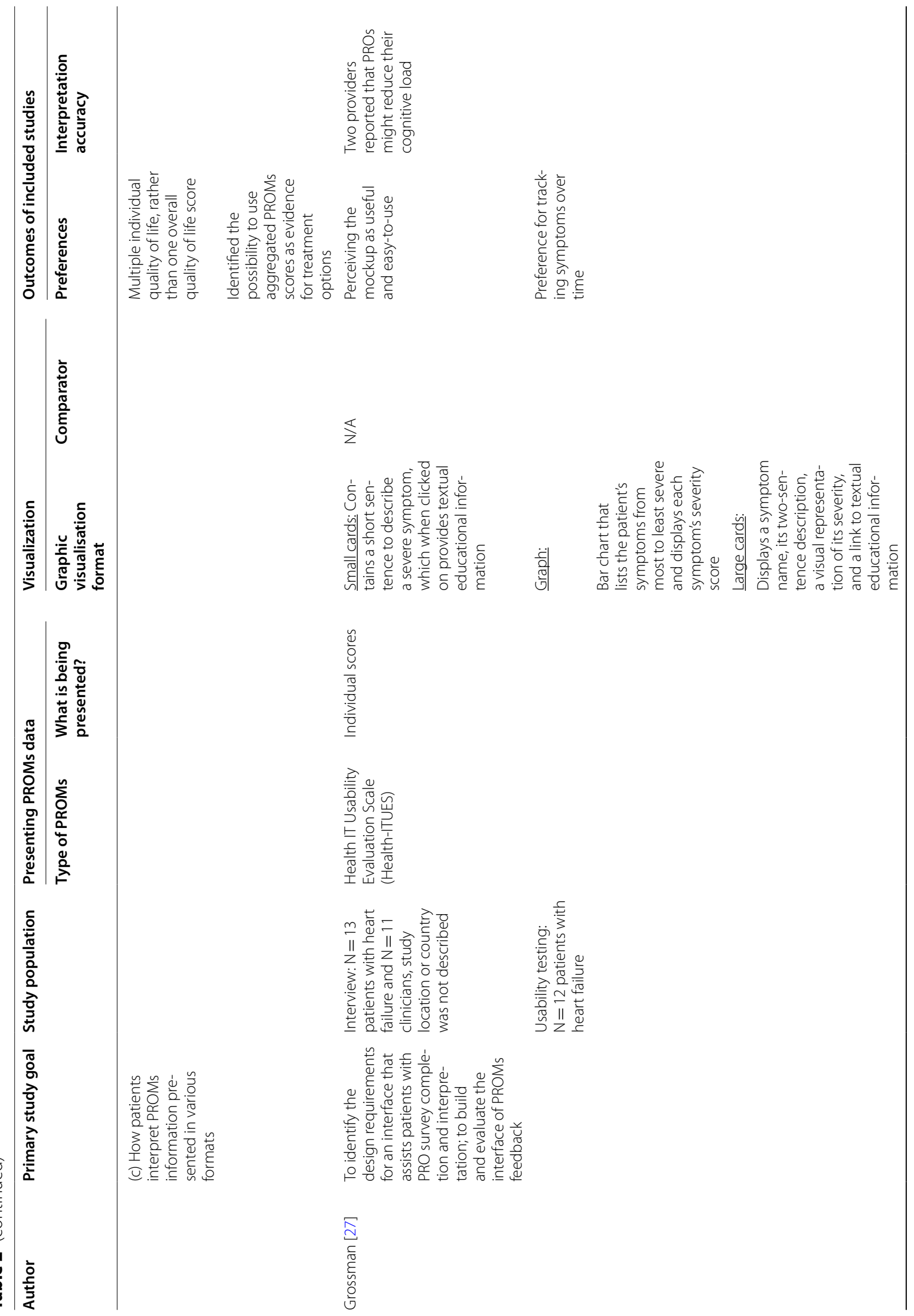




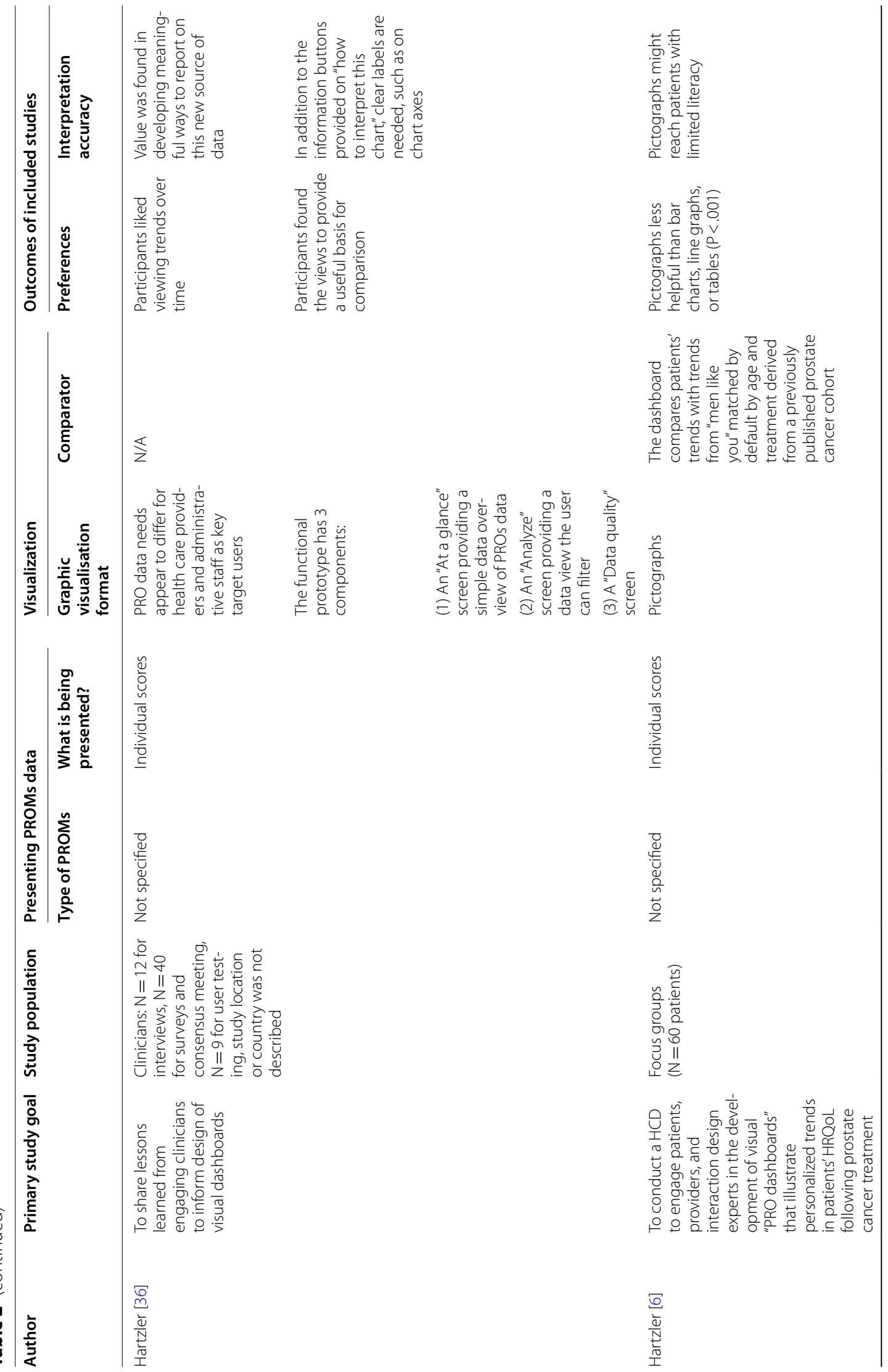




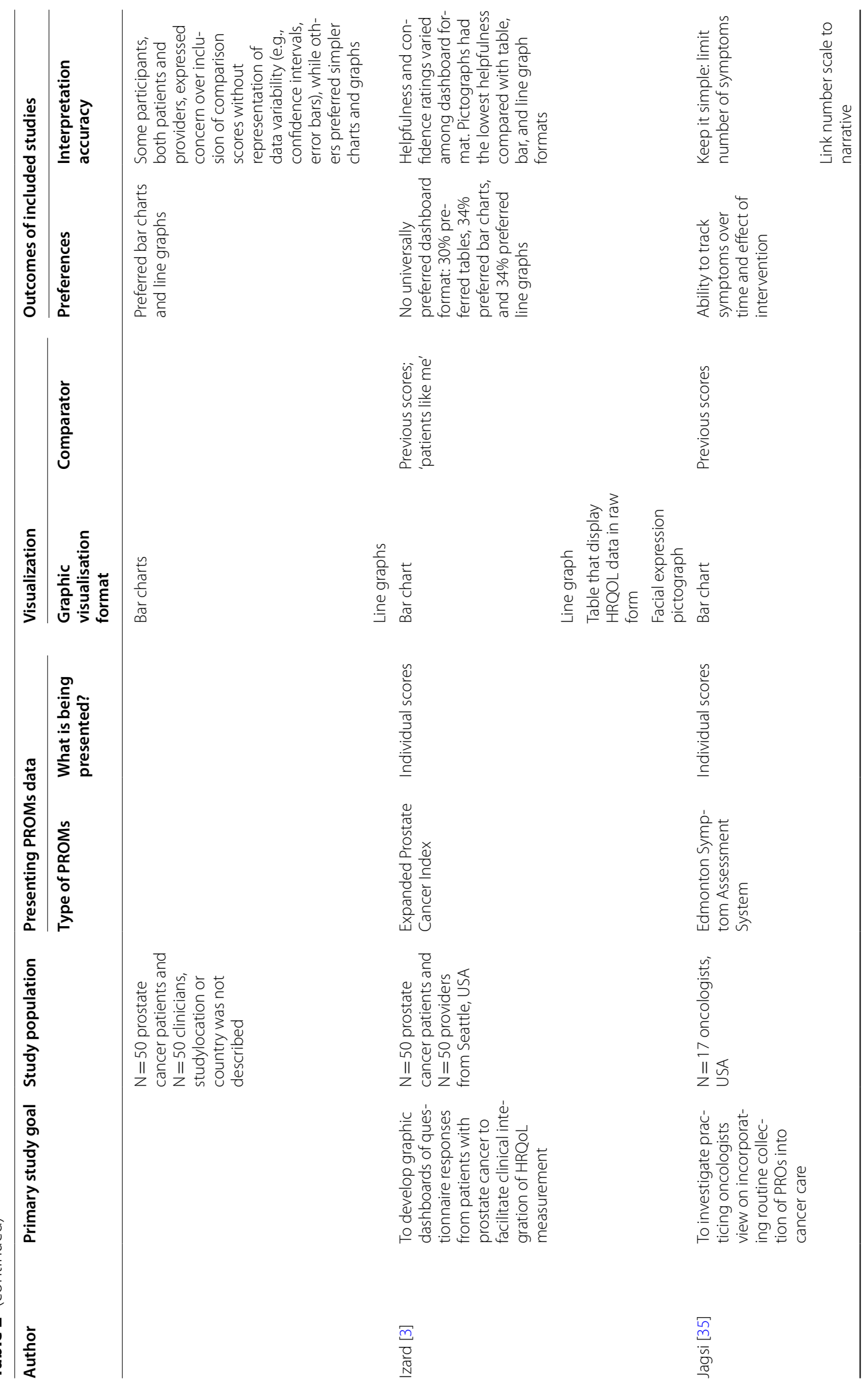




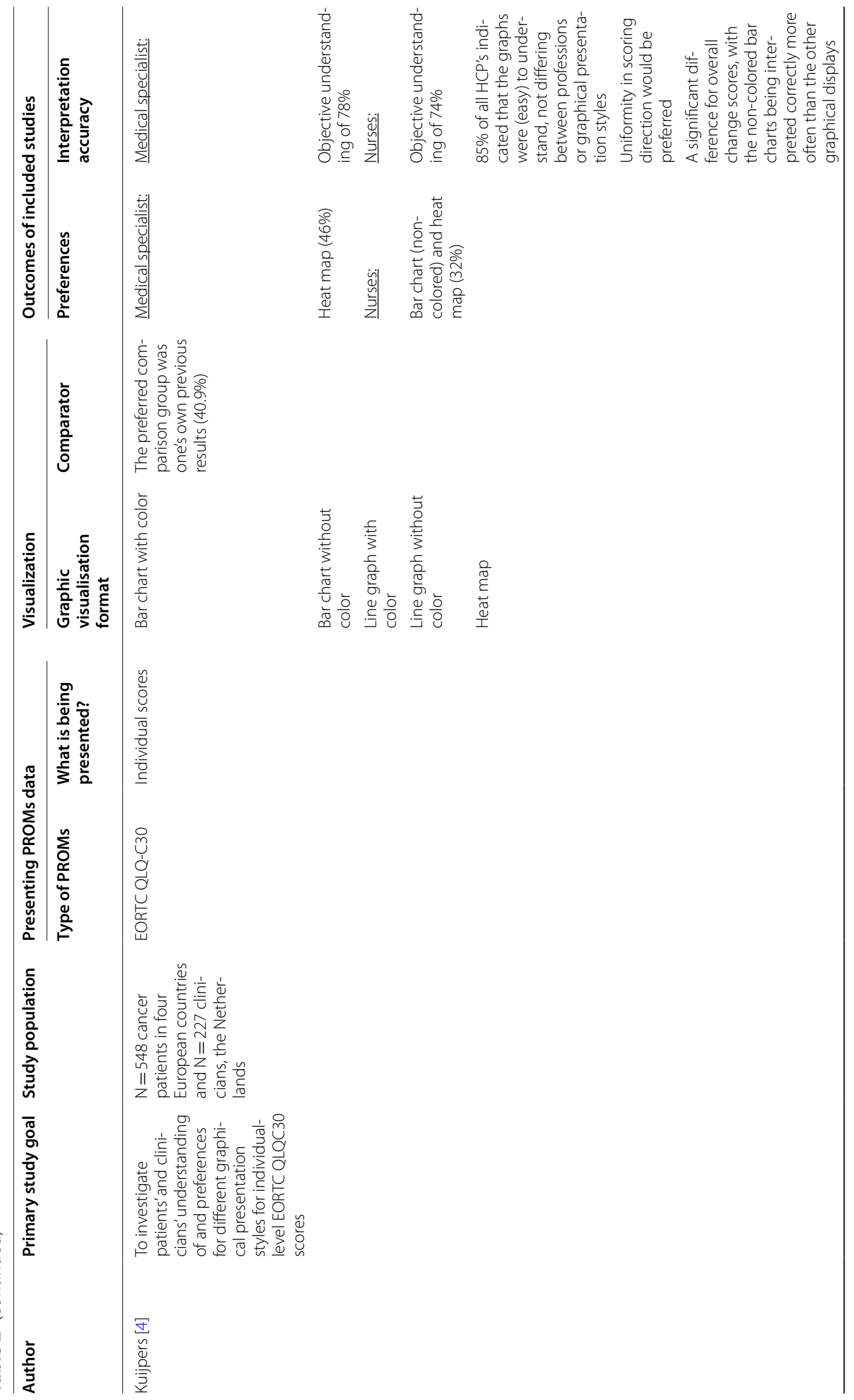




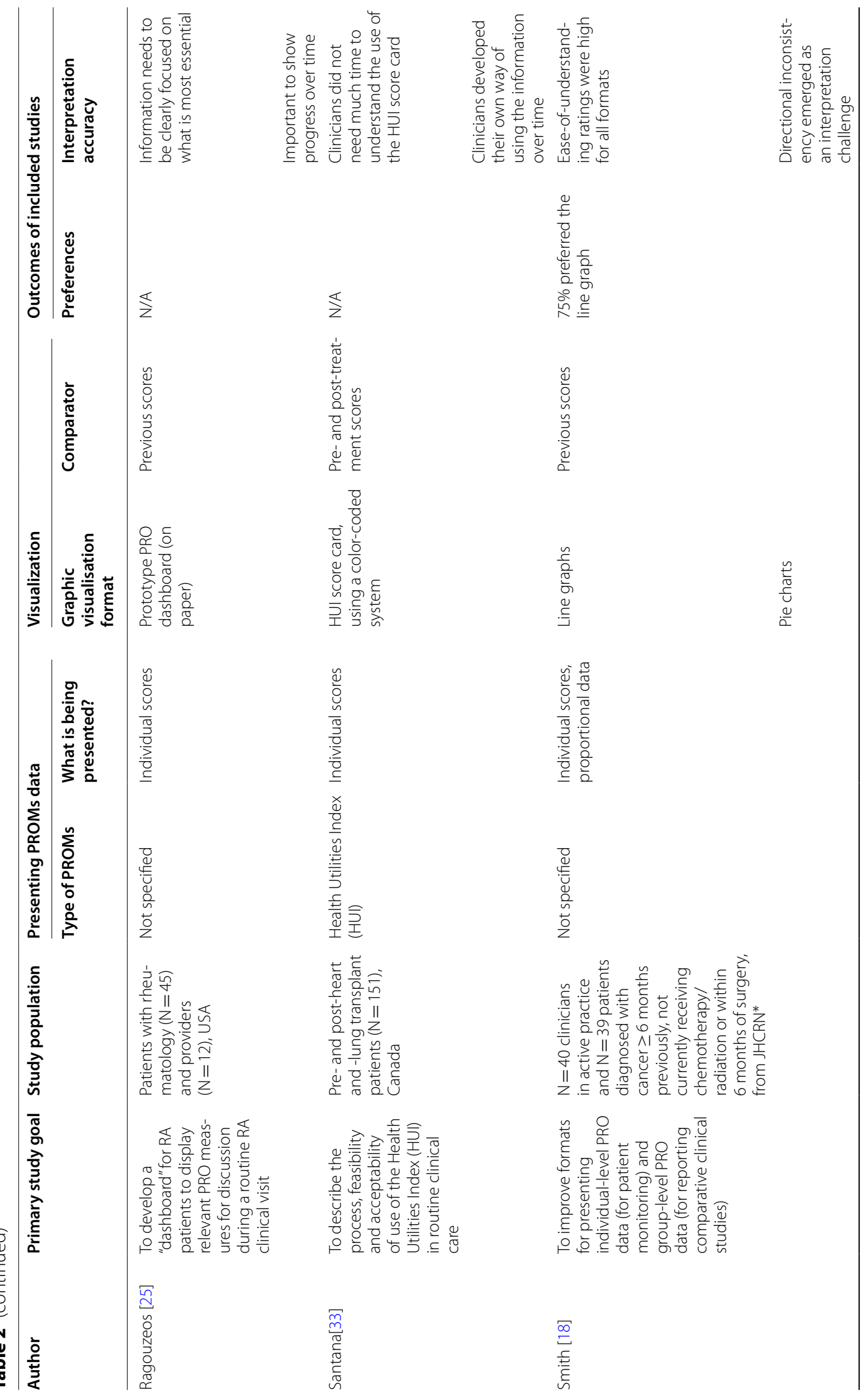




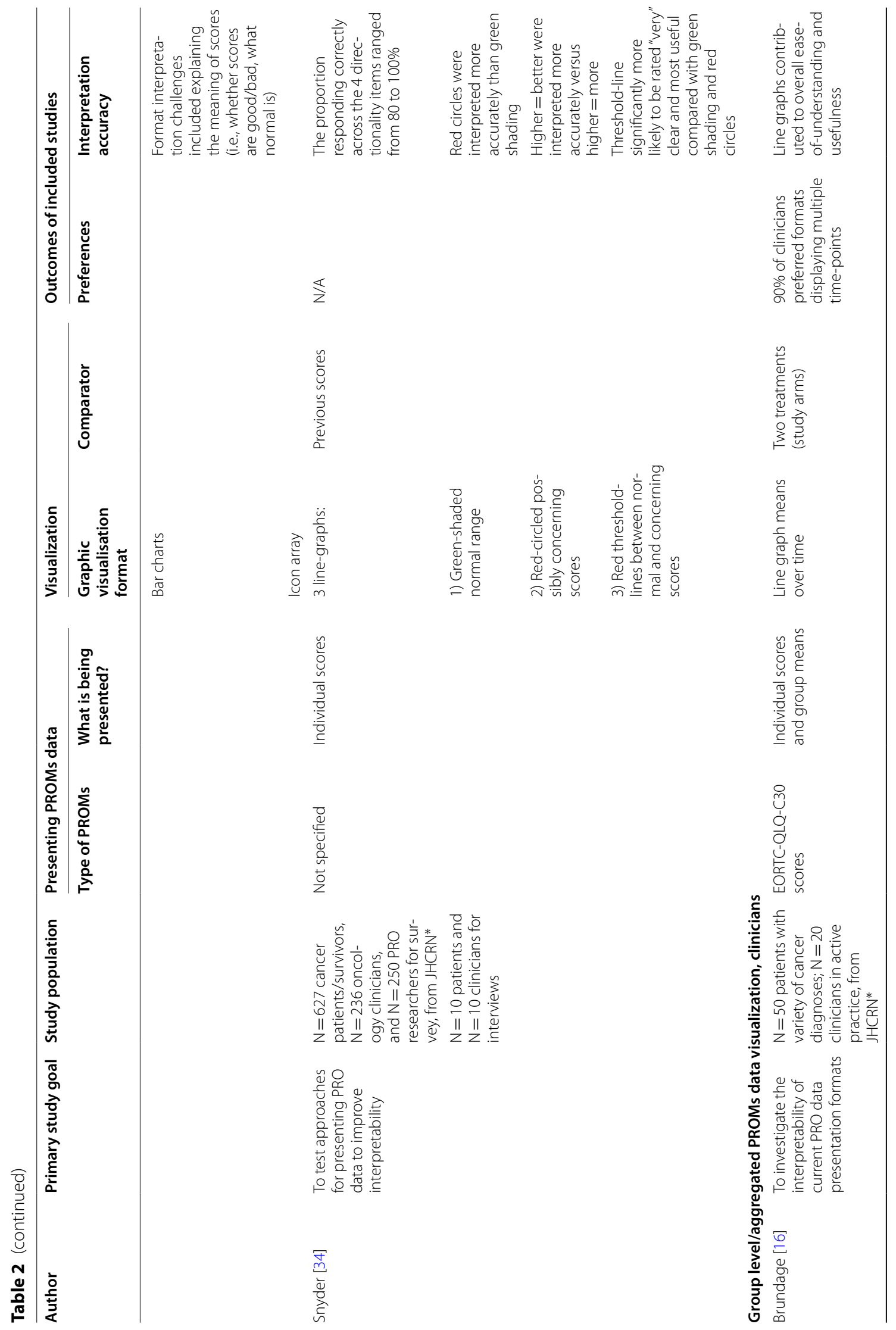




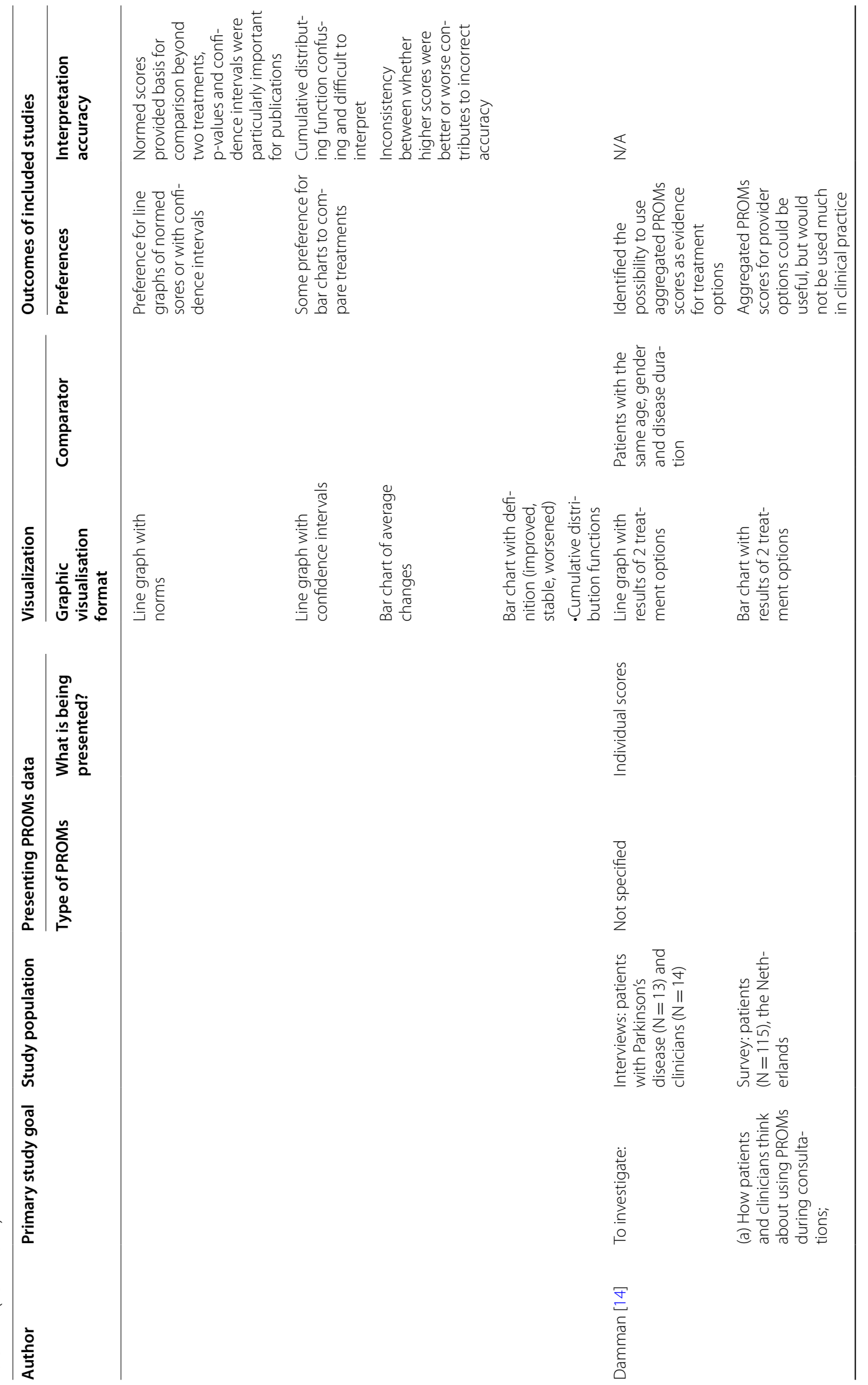




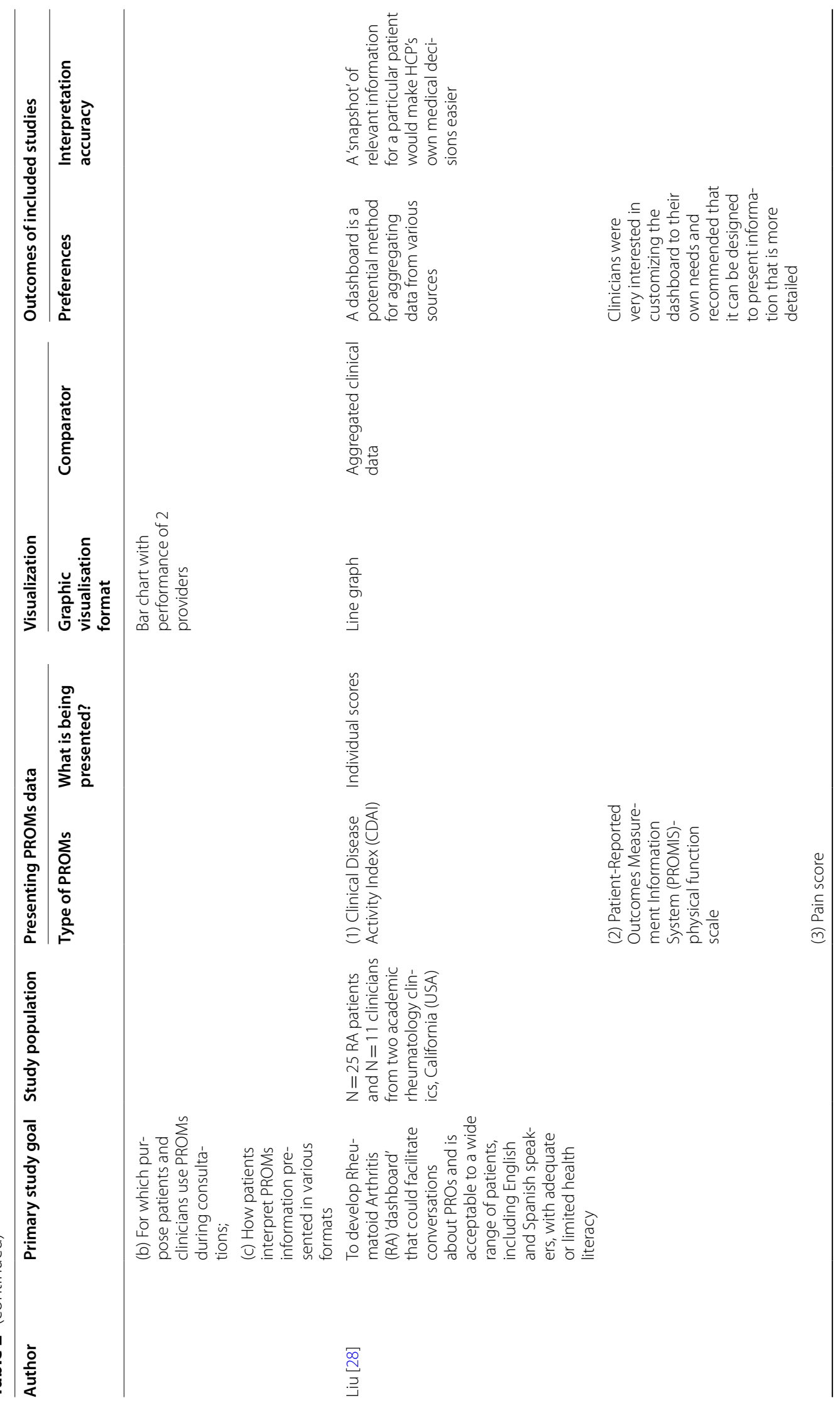




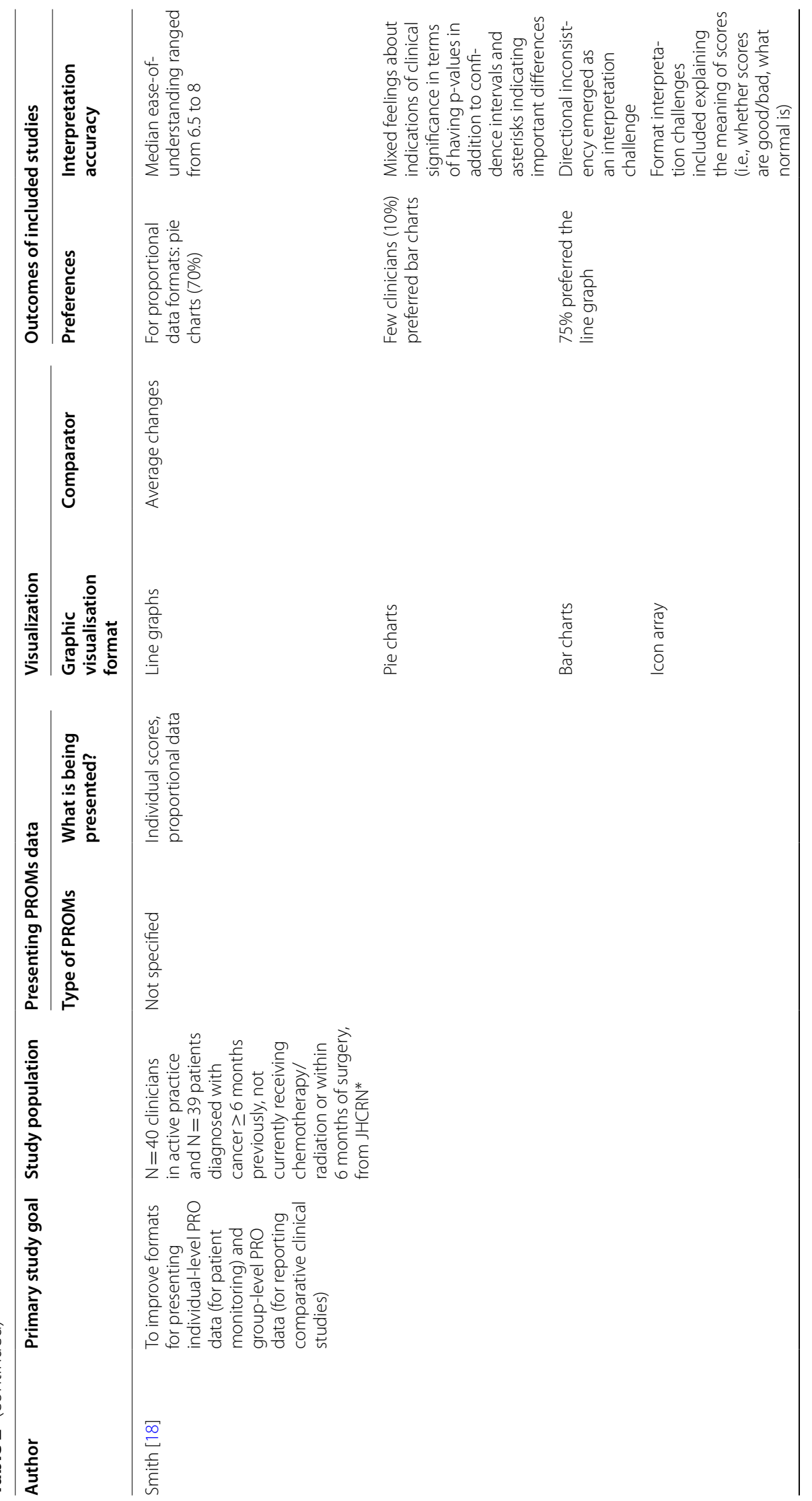




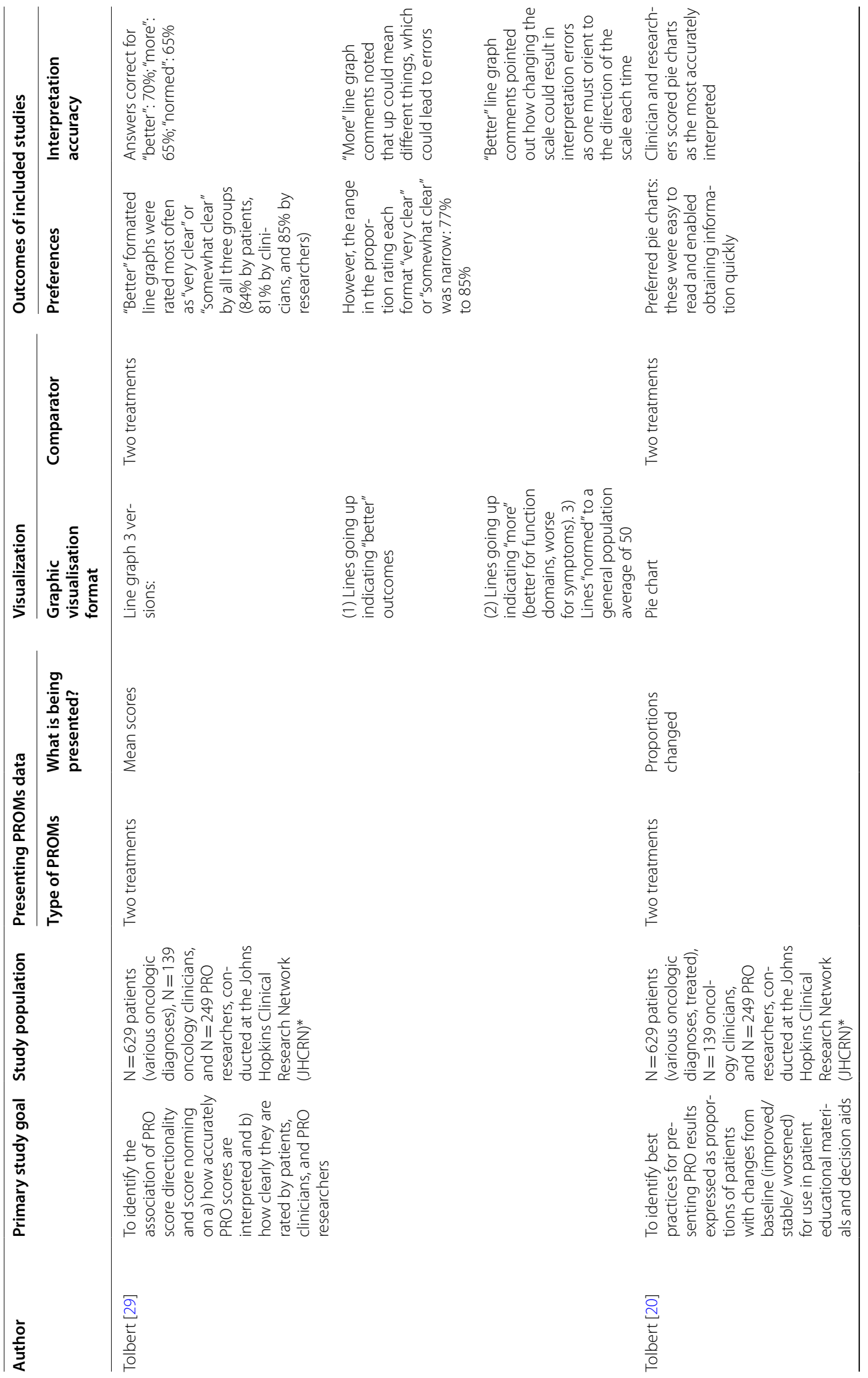




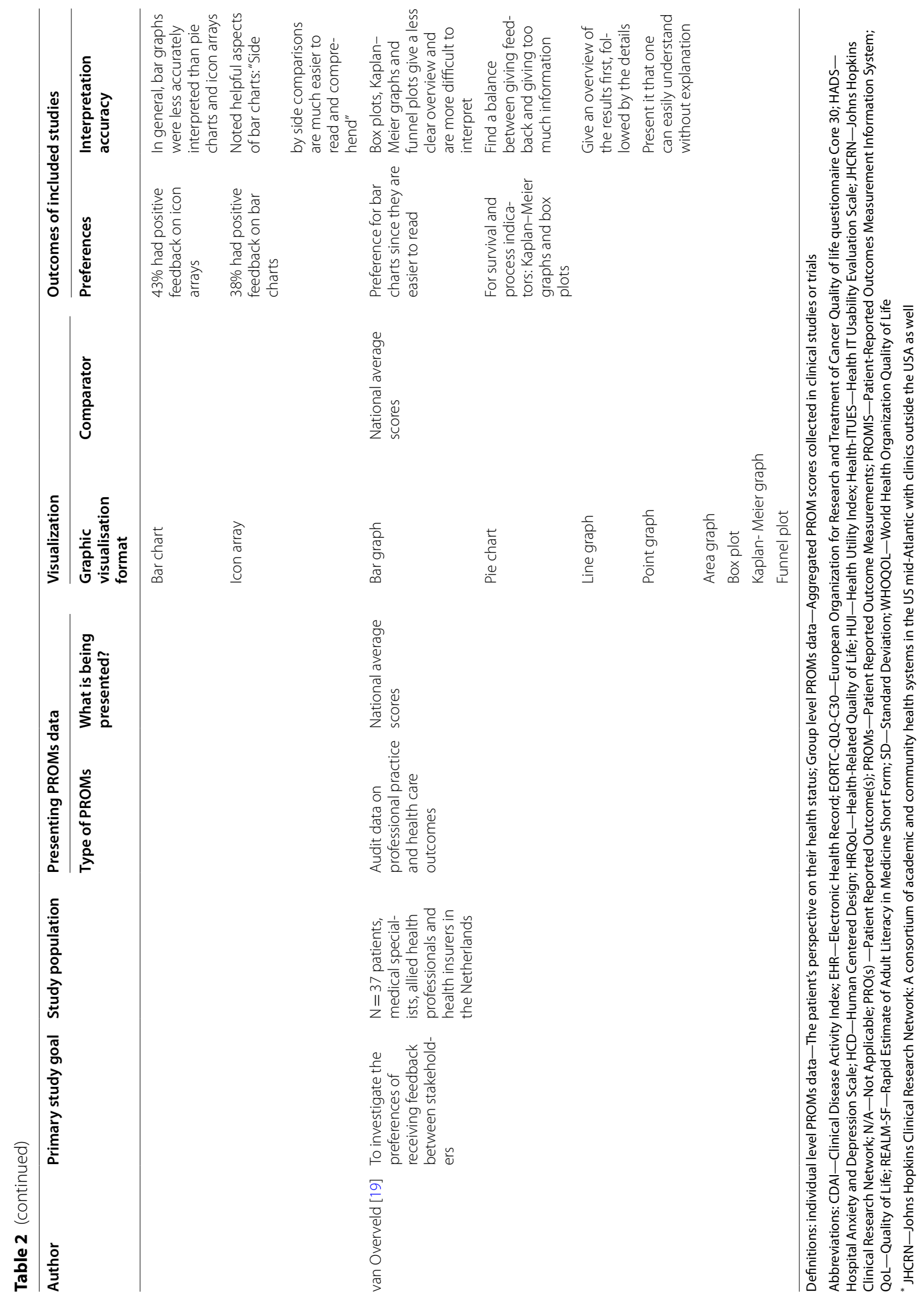




\section{Interpretation accuracy}

Thirteen studies assessed clinicians' interpretation accuracy of graphic visualization formats for individual PROMs level data. Multiple studies showed that clinicians' interpretation accuracy was similar over different graphic visualization formats: both line graphs and bar charts were found easy to understand, and were interpreted accurately $[3,4,18]$. Hartzler et al. [36] reported that among twelve clinicians, respondents generally felt that both line graphs and bar charts provide a useful basis for comparison. Pictographs were reported to be the least helpful for clinicians, although clinicians stated these could be helpful for patients with limited literacy $[3,6]$. Uniformity in directionality of scores could increase clinicians' interpretation accuracy in different graph formats. However, Brundage et al. [37] found that adding asterisks for clinical significance and confidence limits around scores did not contribute to a better interpretation accuracy in clinicians.

\section{Group-level or aggregated PROMs data-clinicians. Preferences}

When presenting aggregated PROMs data, seven studies stated that clinicians mostly compared data between two treatments or compared scores to mean reference population scores. Brundage et al. [16] stated that for comparing treatments, 18 out of 20 clinicians preferred formats displaying multiple time-points, with the highest preference for line graphs with normed scores or confidence intervals, or bar charts. Smith et al. and Tolbert et al. [18, 29 ] describe a preference for line graphs, with over $75 \%$ of included clinicians preferring this graphical format; bar charts were less supported, as only $10 \%$ of 40 clinician participants preferred bar charts [18]. Van Overveld et al. [19] reported however that bar charts were preferred, since they were easy to read. To present proportional data, pie charts were most preferred in 3/8 studies $[18,20,34]$. To meet a variety of preferences, Liu et al. [28] found that a dynamic dashboard gave clinicians the opportunity to customize the formats to their own needs.

\section{Interpretation accuracy}

Six studies assessed clinicians' interpretation accuracy on aggregated data. Line graphs usually contributed to the ease of understanding PROMs scores for clinicians $[16,29]$. Additionally, pie charts with proportional data were most often interpreted accurately by clinicians, for example when pie charts presented a proportional change in outcomes compared to baseline [20]. Clinicians both endorsed and objected that p-values, confidence intervals, and normed scores could contribute to their interpretation accuracy of aggregated PROMs data $[16,18]$. Furthermore, directional consistency, balancing information, and giving feedback, and clear labeling could improve interpretation accuracy $[18,19]$.

Throughout many of the included studies, challenges were described that may affect correctly interpreting visualized PROMs data, such as (1) Directional inconsistency, i.e. a higher functioning score means better health, but a lower symptom score means better health as well [5, 16, 17, 34, 37]; (2) Lack of standardisation rules for interpretation and visualization, that may cause interpretation inaccuracy $[2,25,35]$; (3) The need to designing multiple formats per target group, as no 'one-size-fits-all' solution in graphic visualisation exists for both patients and clinicians $[3,6,28]$; (4) The timing of providing feedback on PROMs visualization, as this affects assessment experience $[14,26]$, and (5) Patients 'opposition to PROMs use in clinical practice $[19,36]$. We summarize the challenges and the proposed solutions for these challenges in Table 3.

\section{How to distinguish clinically relevant PROMs scores}

Clinically alarming scores were put in perspective by comparing current scores to the patients' previous scores or to norm population scores. Ten studies described strategies to distinguish clinically relevant scores in practice [3-5, 16-18, 35]. These studies were conducted internationally among patients with different cancer diagnoses. Most frequently used comparator scores were the patients' own previous PROMs data [3-5, 16-18, 35]. Additionally, four studies used a norm population (i.e.; patients with the same disease) to determine the clinical relevance of scores $[1,5,19,37]$. Patients pointed out that the comparison of their own data with the scores from 'patients like them' (i.e. same sex, and age) was most valuable: it puts the patients functioning in perspective of what is regarded 'normal' [5].

Studies showed different methods of applying color to highlight scores, including: visualizing clinically nonalarming scores in green and clinically alarming scores in red [17]; red and green shading to show undesirable and desirable score ranges, respectively $[4,5,18,25]$; a background fading from red (bottom) to green (top) [1]; traffic light colors (i.e. green, orange, red) [2].

Smith et al. [18] reported that $74 \%$ of patients and $80 \%$ of clinicians preferred green shading of non-alarming scores or red shading of alarming scores. Hildon et al. [2] reported that traffic light colors could improve interpretation accuracy across different graphic visualization formats, since these are universally recognized. Loth et al. [17] reported that $93 \%$ of the studied patients correctly interpreted the meaning of traffic light colors. Nonetheless, PROMs visualization strategies must include a detailed explanatory legend of the meaning and interpretation of colors and scores [4]. 
Table 3 Challenges and factors for improvement to consider when implementing visual individual PROMs feedback in clinical practice

Challenges that may hinder graphic visualization format interpretation

\section{Patients and clinicians}

Directional inconsistency in longitudinal data (i.e., sometimes higher scores can mean better or worse)

Interpretation accuracy of what exact PROMs information is represented in the graphic visualization format

No 'one-size-fits-all' solution

\section{Patients}

Interpretation accuracy of what exact PROMs information is represented in the graphic visualization format

Timing of providing feedback on PROMs visualization

Patients ' opposition to PROMs use in clinical practice

\section{Clinicians}

Interpretation accuracy of what exact PROMs information is represented in the graphic visualization format
Possible factors to improve graphic visualization format interpretation

Make use of standard descriptive labels (consider using 'better*'instead of 'normed ${ }^{* * \prime}$ or 'more $^{* * *}$ for describing directionality of scores) $[34,37]$

Preferred by $79 \%$ of patients and $90 \%$ of clinicians when concerning individual level PROMs data and $100 \%$ of clinicians when concerning group level PROMs data

Consistent use of clear ratings: higher scores are always better results (i.e. in some frequently used PROMs, higher score are better when scores describe functioning, but lower score are better when symptom burden is described. This causes interpretation challenges) [37]

Indicate with an arrow on the $y$-axis which direction means the score is better [16]

Describe directionality by plain text that is understandable despite literacy or education level [5]

Provide detailed information on the meaning of high and low score [17]

Provide an instructive aid for patients and clinicians [2]

Use simple iconography for demonstrating single PROMs values [25]

Use brief definitions of different PROMs domains/values [25]

Limit the number of presented symptoms per graphic visualization format [35]

Make use of a dynamic dashboard, which can display multiple types of visualization strategies. Thereby, you provide users the ability to select a preferred format instead, including the ability to add or remove dashboard elements such as error bars and shading $[3,6,28]$

Developing a clinic-based video tutorial for the dashboard to explain what is shown on the dashboard and how the patient and clinician can customize the dashboard to their needs [28]

Ask patients to prioritize their symptoms, to avoid an overload of information [35]

Provide feedback immediately after assessment, and before consultation, to significantly improve assessment experience when providing combined graphical and tailored text-based feedback $[14,26]$

Ask permission to the patient to receive their own results and/or the results of the general population [19]

Provide information so patients know what PROMs data might show and how their practice might change [36]

Tell patients that data is trustworthy and are handled confidentially $[19,36]$

Do not provide anonymous feedback [19]

Visualize as transparently as possible what type of care is delivered [19]

Eliminate comparison groups or inform comparison group scores with confidence intervals or error bars [3], to better counsel the patients about their score (makes it easier to understand)

Link the PROMs outcome scores (scale in the graphic visualization format) to the meaning of the narrative (i.e.; tell the patient that a higher score on the scale means better functioning) [35]

\footnotetext{
PROMs: patient reported outcome measures

* 'Better' is defined as higher scores indicating "better" outcome

** 'Normed' is defined as normed to the general U.S. population

*** 'More' is defined as higher scores indicating "more" of what was being measured
} 
Other visualization strategies included the use of red circles around important scores-these were interpreted more accurately than green shading [34]. Furthermore, threshold lines across score bars were used to indicate whether scores are better or worse than threshold scores. These visualization techniques pleased $69 \%$ of patients and $70 \%$ of clinicians $[17,18]$. Contrastingly, Snyder et al. [34] reported that a threshold-line was significantly more often rated as 'very clear', and most useful compared to green shading, and red circles. In more detail, another study found that a dotted threshold line was preferred over a solid threshold line to indicate alarming scores in bar charts [5]. Lastly, exclamation points can be used to indicate possibly concerning score changes, which was the preferred method for $79 \%$ of 39 patients and $40 \%$ of 40 clinicians [18].

\section{Discussion}

This systematic review included 25 studies in different healthcare settings throughout Europe, the US, and Canada, that reported about preferences and interpretation accuracy of patients and clinicians for the visualization of PROMs scores. Very few graphical visualization formats for presenting PROMs data could be identified. Overall, a limited amount of literature was found on this matter, which was summarized as follows. For individual level PROMs data, patients and clinicians preferred line graphs and bar charts, since they were considered visually clear. Bar charts were most often interpreted correctly by patients, while clinicians had high interpretation accuracy across all graph formats. For presenting group level PROMs data, pie charts and line graphs were preferred: patients most often interpreted line graphs correctly; for proportional data, clinicians most often accurately interpreted pie charts. To guide clinical interpretation by distinguishing clinically relevant scores, PROMs scores were most often compared to patients' previous scores, followed by comparison to mean norm population scores. Here, correct interpretation can be supported by highlighting patients' clinically alarming scores with colors, and by using threshold lines across score bars or lines, or circles around alarming scores.

Furthermore, we looked into the challenges that may hinder graphic visualization format interpretation. An underlying cause of incorrect interpretation of graphs may be the lack of standardization in rules for interpretation; variability exists in score directionality (e.g. higher scores can either indicate better or worse outcomes), and scaling (e.g. scores ranging from 0 to 100 indicating the worst-to-best possible scores, or scores 'normed' to a defined population). Furthermore, meaningful interpretation of PROMs scores is complicated by the way the statistical and clinical significance of the findings (i.e. thresholds to distinguish clinical importance) are addressed [16, 18, 38, 39]. Therefore, effort must be made to present PROMs scores to patients and clinicians more accurately by: improving directional consistency by making use of standard descriptive labels [34, 37], clear label ratings (i.e. consistent scales ranging from 0-100 [37]), detailed information on the meaning of high and low scores [17], simple iconography [25], and brief definitions to understand what the PROMs scores represent [25]. Furthermore, it was suggested is to visualize only a limited number of symptoms, and to ask patients to prioritize the symptoms they want feedback on [35]. Afore mentioned factors to guide clinical interpretation of graphic visualization formats of PROMs data, were identified as well in a Delphi-consensus study by Snyder et al. [15]. In this study, a panel including 15 doctor or nurse clinicians, 10 participants who identified as patient or caregiver advocates, 12 researchers, and 6 members of journal editorial boards, were asked to review data display issues, and give their perspectives on these issues to develop consensus statements. The authors conclude that implementation of graphic visualization formats of PROMs data have enormous potential to promote patient-centred care, however, it is critical that patients and clinicians understand what PROMs scores mean. More specifically, they recommended to use exceptionally clear labelling, titling, and other annotations to address potential confusion in direction of scores, and warn for mixing score direction in a single display. Furthermore, for conveying score meaning, descriptive labels along the $y$-axis are expected to be helpful [15]. The Setting International Standards of Patient-Reported Outcomes and Quality of Life Endpoints in Cancer Clinical Trials (SISAQoL) guideline [40] reported recommendations on directionality of scale scores similar to Snyder et al. [15]. In order to enhance clinicians' interpretation of PROMs scores they recommended to reduce the number of metrics presented (e.g. a maximum of six bars in bar graphs and 4 lines in line graphs), use coloured arrows (e.g. green for better and red for worse scores) and to accompany more complex displays like funnel plots with a detailed interpretation [40]. This guideline will contribute to standardize rules for interpretation and visualization.

Another suggestion for clarifying PROMs visualisation formats is to develop a dynamic dashboard for PROMs feedback $[3,6,28]$. This way, patients and clinicians are able to change between different graphic visualization formats. We imagine options such as comparing scores to norms or threshold scores, as not all patients may want to do so. Based on the hypothesis that serving individual preferences may facilitate interpretation accuracy, this could improve interpretation of PROMs scores as well. 
However, it should be taken into account that implementing a dynamic dashboard comes with challenges, like access and availability of suitable software and sufficient IT staff to support such a dashboard.

Last, the implementation of PROMs hinges on more factors than visualization of data, starting with the perceived value by patients and clinicians of discussing PROMs during clinical encounters. Nine studies included in our review noted that PROMs were perceived as valuable $[4,5,12,16,25-27,35,36]$, where some studies $(\mathrm{n}=5)$ showed mixed results regarding the usefulness of PROMs [1, 3, 14, 17, 19]. How patients value PROMs may determine the interest in graphic visualization of PROMs. This could have affected the presented results in this review.

This systematic review is limited by the fact that only papers published in English were included. Studies in other languages regarding locally successful implemented feedback of PROMs data might have been missed. Furthermore, in this review, no distinction is made between objective (i.e. does a person actually interpret scores correctly), and subjective (i.e. does the person says he or she interprets scores correctly) interpretation accuracy. Only two included studies made a distinction between the different types of interpretation accuracy [4, 17]; for the other studies, based on the description of the study methods, we believe objective interpretation accuracy was assessed. Therefore, future research may investigate potential differences between actual and perceived interpretation accuracy.

Another potential bias is that for the majority of included studies it remains unclear how questions about preferences and interpretation of the presented visualization format(s) were framed to the study population. Besides, study participants may have had different background knowledge about graphic visualization formats, presentation of formats, and the content of PROMs data. This could confound our findings about how data and graphs were interpret, understood, and valued.

Furthermore, this review did not consider different levels of patients' health literacy, since this information was not presented in the majority of articles reviewed. Patients with a higher level of health literacy can have different preferences compared to patients with lower levels of health literacy [3]. Some patients with limited health literacy may not understand the longitudinal nature of data from left to right nor the temporal connection between different graphical elements [28]. Therefore, healthy literacy should be included as factor in studies dealing with data interpretation, for example measured through the use of the validated Health Literacy Questionnaire (HLQ) [41]. Furthermore, health literacy can differ among age, gender, and/or education level and therefore should be studied among these separate groups of patients in relation to PROMs visualization $[42,43]$.

In conclusion, there was no predominant graphical visualization format approach in terms of preferences or interpretation accuracy for both patients and clinicians. To guide the clinical interpretation of scores during clinical encounters, PROMs scores can be compared to patients' previous scores or mean scores from a norm population, or compared to comparator scores or score thresholds. Furthermore, the use of colors, threshold lines, or circles around alarming scores can visualize the clinical meaning of PROMs scores that have been compared to previous scores, norms, thresholds, or reference populations. Finally, detailed clarification of each graph may be essential for accurate interpretation. Visualization strategies should therefore include descriptions of PROMs directionality as well as standard descriptive labels, brief definitions, and presentation of a limited number of symptoms in a graph.

\section{Abbreviations}

CASP: Critical appraisal skills programme; CDAl: Clinical disease activity index; HER: Electronic Health Record; EORTC-QLQ-C30: European organization for research and treatment of cancer quality of life questionnaire Core 30; HADS: Hospital anxiety and depression scale; HCD: Human centered design; HRQoL: Health-related quality of life; HUI: Health utility index; Health-ITUES: Health IT usability evaluation scale; HLQ: Health literacy questionnaire; JHCRN: Johns Hopkins clinical research network; N/A: Not applicable; PRISMA: Preferred reporting items for systematic reviews and meta-analysis; PROMs: Patient reported outcome measurements; PROMIS: Patient-reported outcomes measurement information system; QoL: Quality of life; REALM-SF: Rapid estimate of adult literacy in medicine short form; SISAQOL: Setting international standards of patient-reported outcomes and quality of life endpoints in cancer clinical trials; SD: Standard deviation; USA: United States of America; WHOQOL: World health organization quality of life.

\section{Supplementary Information}

The online version contains supplementary material available at https://doi. org/10.1186/s41687-022-00424-3.

Additional file 1: Table S1. Search strategy for MEDLINE (accessed through PubMed), Embase (accessed through Ovid Platform), PsycINFO (accessed through Ovid Platform) and CINAHL.

\section{Acknowledgements}

Not applicable.

\section{Authors' contributions}

EA, IF: Conceptualization, article screening, data analysis, editing, writing. KdL: conceptualization, literature search, data analysis, editing, reviewing, writing. IW, LvdP: conceptualization, editing, reviewing, writing. EW: literature search, reviewing method section, PRISMA. SS, MW, IvdP: conceptualization, reviewing, writing. All authors read and approved the final manuscript.

Funding

Not applicable. 


\section{Availability of data and materials}

Data sharing is not applicable to this article as no datasets were generated or analysed during the current study.

\section{Declarations}

\section{Ethics approval and consent to participate}

Not applicable.

\section{Consent for publication}

Not applicable.

\section{Competing interests}

The authors declare that they have no competing interests.

\section{Author details}

${ }^{1}$ Department of Psychosocial Research, Division of Psychosocial Research and Epidemiology, The Netherlands Cancer Institute, Amsterdam, The Netherlands. ${ }^{2}$ Department for Health Evidence, Radboudumc, Nijmegen, The Netherlands. ${ }^{3}$ Library and Scientific Information Department, The Netherlands Cancer Institute, Amsterdam, The Netherlands. ${ }^{4}$ Department of Psychology, University of Amsterdam, Amsterdam, The Netherlands. ${ }^{5}$ Department of Surgical Oncology, Antoni Van Leeuwenhoek, Amsterdam, The Netherlands. ${ }^{6}$ Department of Biomedical Data Sciences, Leiden University Medical Center, Leiden, The Netherlands. ${ }^{7}$ Department of Research and Development, Netherlands Comprehensive Cancer Organization, Utrecht, The Netherlands. ${ }^{8}$ Department of Medical and Clinical Psychology, Center of Research On Psychological and Somatic Disorders (CoRPS), Tilburg University, Tilburg, The Netherlands.

Received: 27 July 2021 Accepted: 16 February 2022

Published online: 03 March 2022

\section{References}

1. Fischer Kl, De Faoite D, Rose M (2020) Patient-reported outcomes feedback report for knee arthroplasty patients should present selective information in a simple design - findings of a qualitative study. J Patient Rep Outcomes 4(1):6

2. Hildon Z, Allwood D, Black N (2012) Making data more meaningful: patients' views of the format and content of quality indicators comparing health care providers. Patient Educ Couns 88(2):298-304

3. Izard J, Hartzler A, Avery DI, Shih C, Dalkin BL, Gore JL (2014) Usercentered design of quality of life reports for clinical care of patients with prostate cancer. Surgery 155(5):789-796

4. Kuijpers W, Giesinger JM, Zabernigg A, Young T, Friend E, Tomaszewska IM et al (2016) Patients' and health professionals' understanding of and preferences for graphical presentation styles for individual-level EORTC QLQ-C30 scores. Qual Life Res 25(3):595-604

5. Oerlemans S, Arts LP, Horevoorts NJ, van de Poll-Franse LV (2017) "Am I normal?" The Wishes of patients with lymphoma to compare their patient-reported outcomes with those of their peers. J Med Internet Res. 19(8):e288.

6. Hartzler AL, Izard JP, Dalkin BL, Mikles SP, Gore JL (2016) Design and feasibility of integrating personalized PRO dashboards into prostate cancer care. J Am Med Inform Assoc 23(1):38-47

7. Snyder C, Brundage M, Rivera YM, Wu AW (2019) A PRO-cision medicine methods toolkit to address the challenges of personalizing cancer care using patient-reported outcomes: introduction to the supplement. Med Care. 57:51-s7

8. Snyder CF, Blackford AL, Aaronson NK, Detmar SB, Carducci MA, Brundage MD et al (2011) Can patient-reported outcome measures identify cancer patients' most bothersome issues? J Clin Oncol 29(9):1216-1220

9. Berry DL, Blumenstein BA, Halpenny B, Wolpin S, Fann JR, Austin-Seymour $M$ et al (2011) Enhancing patient-provider communication with the electronic self-report assessment for cancer: a randomized trial. J Clin Oncol 29(8):1029-1035

10. Yang LY, Manhas DS, Howard AF, Olson RA (2018) Patient-reported outcome use in oncology: a systematic review of the impact on patientclinician communication. Supp Care Cancer 26(1):41-60
11. Chen J, Ou L, Hollis SJ (2013) A systematic review of the impact of routine collection of patient reported outcome measures on patients, providers and health organisations in an oncologic setting. BMC Health Serv Res 13:211

12. Brundage M, Leis A, Bezjak A, Feldman-Stewart D, Degner L, Velji K et al (2003) Cancer patients' preferences for communicating clinical trial quality of life information: a qualitative study. Qual Life Res 12(4):395-404

13. Bantug ET, Coles T, Smith KC, Snyder CF, Rouette J, Brundage MD et al (2016) Graphical displays of patient-reported outcomes (PRO) for use in clinical practice: What makes a pro picture worth a thousand words? Patient Educ Couns 99(4):483-490

14. Damman OC, Verbiest MEA, Vonk SI, Berendse HW, Bloem BR, de Bruijne MC et al (2019) Using PROMs during routine medical consultations: The perspectives of people with Parkinson's disease and their health professionals. Health Expect 22(5):939-951

15. Snyder C, Smith K, Holzner B, Rivera YM, Bantug E, Brundage M et al (2019) Making a picture worth a thousand numbers: recommendations for graphically displaying patient-reported outcomes data. Qual Life Res 28(2):345-356

16. Brundage MD, Smith KC, Little EA, Bantug ET, Snyder CF (2015) Communicating patient-reported outcome scores using graphic formats: results from a mixed-methods evaluation. Qual Life Res 24(10):2457-2472

17. Loth FL, Holzner B, Sztankay M, Bliem HR, Raoufi S, Rumpold G et al (2016) Cancer patients' understanding of longitudinal EORTC QLQ-C30 scores presented as bar charts. Patient Educ Couns 99(12):2012-2017

18. Smith KC, Brundage MD, Tolbert E, Little EA, Bantug ET, Snyder CF et al (2016) Engaging stakeholders to improve presentation of patient-reported outcomes data in clinical practice. Supp Care Cancer 24(10):4149-4157

19. van Overveld LFJ, Takes RP, Vijn TW, Braspenning JCC, de Boer JP, Brouns JJA et al (2017) Feedback preferences of patients, professionals and health insurers in integrated head and neck cancer care. Health Expect 20(6):1275-1288

20. Tolbert E, Brundage M, Bantug E, Blackford AL, Smith K, Snyder C et al (2019) In proportion: approaches for displaying patient-reported outcome research study results as percentages responding to treatment. Qual Life Res 28(3):609-620

21. Basch E, Barbera L, Kerrigan CL, Velikova G (2018) Implementation of patient-reported outcomes in routine medical care. Am Soc Clin Oncol Educ Book 38:122-134

22. Shamseer L, Moher D, Clarke M, Ghersi D, Liberati A, Petticrew M, et al (2015) Preferred reporting items for systematic review and meta-analysis protocols (PRISMA-P) 2015: elaboration and explanation. Bmj.350:g7647

23. Bramer WM, Giustini D, de Jonge GB, Holland L, Bekhuis T (2016) Deduplication of database search results for systematic reviews in EndNote. J Med Libr Assoc 104(3):240-243

24. Nadelson S, Nadelson LS (2014) Evidence-based practice article reviews using CASP tools: a method for teaching EBP. Worldviews Evid Based Nurs 11(5):344-346

25. Ragouzeos D, Gandrup J, Berrean B, Li J, Murphy M, Trupin L et al (2019) "Am I OK?" using human centered design to empower rheumatoid arthritis patients through patient reported outcomes. Patient Educ Couns 102(3):503-510

26. Geerards D, Pusic A, Hoogbergen M, van der Hulst R, Sidey-Gibbons C (2019) Computerized Quality of Life Assessment: A Randomized Experiment to Determine the Impact of Individualized Feedback on Assessment Experience. J Med Internet Res.21(7):e12212.

27. Grossman LV, Feiner SK, Mitchell EG, Masterson Creber RM (2018) Leveraging patient-reported outcomes using data visualization. Appl Clin Inform 9(3):565-575

28. Liu LH, Garrett SB, Li J, Ragouzeos D, Berrean B, Dohan D et al (2020) Patient and clinician perspectives on a patient-facing dashboard that visualizes patient reported outcomes in rheumatoid arthritis. Health Expect 23(4):846-859

29. Tolbert E, Brundage M, Bantug E, Blackford AL, Smith K, Snyder C et al (2018) Picture this: presenting longitudinal patient-reported outcome research study results to patients. Med Decis Making 38(8):994-1005

30. Brundage M, Feldman-Stewart D, Leis A, Bezjak A, Degner L, Velji K et al (2005) Communicating quality of life information to cancer patients: a study of six presentation formats. J Clin Oncol 23(28):6949-6956 
31. Brundage MD, Smith KC, Little EA, Bantug ET, Snyder CF (2015) Board PRODPSA. Communicating patient-reported outcome scores using graphic formats: results from a mixed-methods evaluation. Qual Life Res. 24(10):2457-72

32. McNair AG, Brookes ST, Davis CR, Argyropoulos M, Blazeby JM (2010) Communicating the results of randomized clinical trials: do patients understand multidimensional patient-reported outcomes? J Clin Oncol 28(5):738-743

33. Santana MJ, Feeny DH (2009) Using the health utilities index in routine clinical care: process, feasibility, and acceptability: a randomized controlled trial. Patient 2(3):159-167

34. Snyder CF, Smith KC, Bantug ET, Tolbert EE, Blackford AL, Brundage MD et al (2017) What do these scores mean? Presenting patient-reported outcomes data to patients and clinicians to improve interpretability. Cancer 123(10):1848-1859

35. Jagsi R, Chiang A, Polite BN, Medeiros BC, McNiff K, Abernethy AP et al (2013) Qualitative analysis of practicing oncologists' attitudes and experiences regarding collection of patient-reported outcomes. J Oncol Pract 9(6):e290-e297

36. Hartzler AL, Chaudhuri S, Fey BC, Flum DR, Lavallee D (2015) Integrating Patient-Reported Outcomes into Spine Surgical Care through Visual Dashboards: Lessons Learned from Human-Centered Design. EGEMS (Wash DC) 3(2):1133

37. Brundage M, Blackford A, Tolbert E, Smith K, Bantug E, Snyder C et al (2018) Presenting comparative study PRO results to clinicians and researchers: beyond the eye of the beholder. Qual Life Res 27(1):75-90

38. Brundage M, Bass B, Jolie R, Foley K (2011) A knowledge translation challenge: clinical use of quality of life data from cancer clinical trials. Qual Life Res 20(7):979-985

39. Snyder CF, Aaronson NK (2009) Use of patient-reported outcomes in clinical practice. Lancet 374(9687):369-370

40. Hancock SL, Ryan OF, Marion V, Kramer S, Kelly P, Breen S, et al (2020) Feedback of patient-reported outcomes to healthcare professionals for comparing health service performance: a scoping review. BMJ Open. 10(11):e038190

41. Osborne RH, Batterham RW, Elsworth GR, Hawkins M, Buchbinder R (2013) The grounded psychometric development and initial validation of the Health Literacy Questionnaire (HLQ). BMC Public Health 13:658

42. Williams MV, Davis T, Parker RM, Weiss BD (2002) The role of health literacy in patient-physician communication. Fam Med 34(5):383-389

43. Clouston SAP, Manganello JA, Richards M (2017) A life course approach to health literacy: the role of gender, educational attainment and lifetime cognitive capability. Age Ageing 46(3):493-499

\section{Publisher's Note}

Springer Nature remains neutral with regard to jurisdictional claims in published maps and institutional affiliations.

\section{Submit your manuscript to a SpringerOpen ${ }^{\circ}$ journal and benefit from:}

- Convenient online submission

- Rigorous peer review

- Open access: articles freely available online

- High visibility within the field

- Retaining the copyright to your article 